\title{
Functional Programming for Compiling and Decompiling Computer-Aided Design
}

CHANDRAKANA NANDI, University of Washington, USA

JAMES R. WILCOX, University of Washington, USA

PAVEL PANCHEKHA, University of Washington, USA

TAYLOR BLAU, University of Washington, USA

DAN GROSSMAN, University of Washington, USA

ZACHARY TATLOCK, University of Washington, USA

Desktop-manufacturing techniques like 3D printing are increasingly popular because they reduce the cost and complexity of producing customized objects on demand. Unfortunately, the vibrant communities of early adopters, often referred to as "makers," are not well-served by currently available software pipelines. Users today must compose idiosyncratic sequences of tools which are typically superposed variants of proprietary software originally designed for expert specialists.

This paper proposes fundamental programming-languages techniques to bring improved rigor, reduced complexity, and new functionality to the computer-aided design (CAD) software pipeline for applications like 3D-printing. Compositionality, denotational semantics, compiler correctness, and program synthesis all play key roles in our approach, starting from the perspective that solid geometry is a programming language.

Specifically, we define a purely functional language for $\mathrm{CAD}$ called $\lambda \mathrm{CAD}$ and a polygon surface-mesh intermediate representation. We then define denotational semantics of both languages to 3D solids and a compiler from CAD to mesh accompanied by a proof of semantics preservation. We illustrate the utility of this foundation by developing a novel synthesis algorithm based on evaluation contexts to "reverse compile" difficult-to-edit meshes downloaded from online maker communities back to more-editable CAD programs. All our prototypes have been implemented in OCaml to enable further exploration of functional programming for desktop manufacturing.

CCS Concepts: • Theory of computation $\rightarrow$ Program semantics; $\bullet$ Software and its engineering $\rightarrow$ Compilers;

Additional Key Words and Phrases: language design, denotational semantics, program synthesis, 3D printing

\section{ACM Reference Format:}

Chandrakana Nandi, James R. Wilcox, Pavel Panchekha, Taylor Blau, Dan Grossman, and Zachary Tatlock. 2018. Functional Programming for Compiling and Decompiling Computer-Aided Design. Proc. ACM Program. Lang. 2, ICFP, Article 99 (September 2018), 31 pages. https://doi.org/10.1145/3236794

\section{INTRODUCTION}

Democratized computer-aided manufacturing has made available-at modest cost-design and fabrication capabilities that were previously reserved for large-scale commercial applications.

Authors' addresses: Chandrakana Nandi, University of Washington, USA, cnandi@cs.washington.edu; James R. Wilcox, University of Washington, USA, jrw12@cs.washington.edu; Pavel Panchekha, University of Washington, USA, pavpan@cs. washington.edu; Taylor Blau, University of Washington, USA, ttaylorr@cs.washington.edu; Dan Grossman, University of Washington, USA, djg@cs.washington.edu; Zachary Tatlock, University of Washington, USA, ztatlock@cs.washington.edu.

This work is licensed under a Creative Commons Attribution 4.0 International License.

(C) 2018 Copyright held by the owner/author(s).

2475-1421/2018/9-ART99

https://doi.org/10.1145/3236794

Proc. ACM Program. Lang., Vol. 2, No. ICFP, Article 99. Publication date: September 2018. 


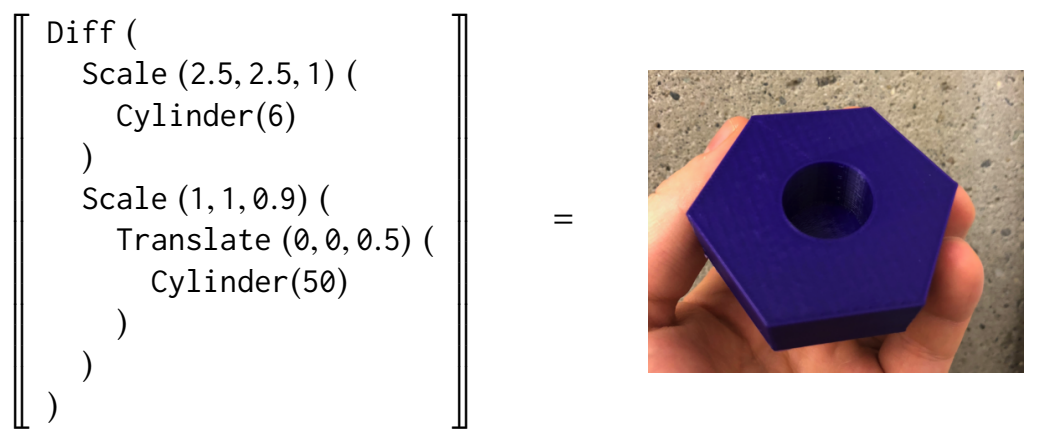

A computer-aided design program denotes a geometric object.

Desktop-class 3D printers, laser cutters, and computer numerical control mills affordably enable educators, hobbyists, and researchers to rapidly prototype designs, manufacture tool parts, and even create custom prostheses [The Future 2018]. 3D-printers in particular are now standard tools in maker communities and may some day replace the need for small-scale manufacturing much as conventional printers fundamentally changed the role of commercial printing shops.

However, despite the wide availability of hardware components at much lower costs than ever before, the corresponding software pipeline does not sufficiently support even tech-savvy early adopters. For democratized manufacturing techniques to reach their full potential, makers must be able to design and manufacture a wide variety of objects on demand.

The current state of tools in this space expects users to compose idiosyncratic CAD packages that are incompatible and whose interfaces are not clearly specified. Together with the fact that most of these tools are also proprietary, it makes the design experience for novice users and hobbyists unnecessarily awkward. The lack of specification also hampers efforts to build other tools that can make $\mathrm{CAD}$ programming more accessible to amateur enthusiasts. For example, it would benefit users to have tools for debugging their designs before printing to avoid waste of time and material, optimizing them to find an equivalent but simpler program, doing program analysis to detect violations of various geometric and physical properties, or synthesizing CAD programs for them so that they do not have to program from scratch.

This paper takes preliminary steps toward addressing these challenges from a programminglanguages perspective. Our first contribution is based on the insight that the desktop manufacturing pipeline is inherently compositional and functional in nature. We view this pipeline as a compilation task by modeling 3D solid geometry as a purely functional programming language equipped with a natural and tangible denotation to 3D solids. To relate this high-level CAD language to intermediate mesh representations, we formalize the popular STL mesh format [Grimm 2004] as a low-level language and define a meaning-preserving compiler from $\mathrm{CAD}$ programs to meshes. We have designed and implemented a prototype of our declarative CAD language called $\lambda C A D$ that supports $3 \mathrm{D}$ primitives such as cubes, spheres, and cylinders; affine transformations such as translation, scaling, and rotation; and binary or constructive solid geometry (CSG) operations such as difference, intersection, and union. $\lambda \mathrm{CAD}$ also supports standard functional features such as let bindings, functions, recursion, and conditionals.

Having developed this foundation, we define compiler correctness in terms of solid geometry, and provide a proof that our compiler is correct under this definition. Our approach toward formalizing CAD and STL then leads to the other main contribution in this paper-the first synthesis algorithm 
to our knowledge that converts meshes back into CAD programs, which we view as a reverse compilation task. Given a surface mesh, our algorithm finds a CAD program, which, when compiled, gives that mesh.

It turns out that reverse compilation may have the potential to solve a key problem for the current state of the 3D-printer enthusiast community: Many hobbyists and makers lack the requisite expertise to translate their ideas into CAD programs from scratch. To overcome this barrier, they often download and print existing designs from online communities [GrabCAD 2018; Thingiverse 2018b] where experts share their work freely. These repositories distribute designs as polygon meshes instead of $\mathrm{CAD}$ programs because $\mathrm{CAD}$ does not have standardized representations, so meshes, in the standard STL format, are the cross-platform distribution language. However, users are rarely able to customize designs shared as meshes [Alcock et al. 2016; Hudson et al. 2016]. Mesh modification tools [Meshmixer 2018] are useful for only some types of low-level modifications, and even then are difficult to use because they can easily break the model, thus making it invalid and unprintable. In large part, this is because mesh models have had all high-level design information "compiled away," analogous to how program binaries have had high-level operations compiled down to primitive machine operations.

Reverse compilation extracts high-level structural information from the design that enables rich edits to the design, which would otherwise require tedious low-level edits directly on the surface mesh. Our algorithm combines basic computational geometry with program synthesis to elegantly search the space of possible CAD programs. It repurposes the traditional PL machinery of evaluation contexts to guide the search of the synthesis algorithm toward the lowest-cost (ideally, the most "human-editable") CAD program.

We have implemented the synthesis algorithm in a prototype tool called ReIncarnate. We detail three case studies that demonstrate our approach on samples selected from Thingiverse. They cover three popular applications of desktop 3D printing: machine tools, household objects, and aesthetic items designed by hobbyists. We give examples of modifications that are intractable to make in mesh models but become simple at the CAD level.

Our implementation of the compiler and synthesis prototypes consists of about 20000 LOC of $\mathrm{OCaml}^{1}$. As Section 4.3 describes, we leverage several features of OCaml to achieve simplicity and modularity, while also laying the groundwork for further evaluation such as differential testing. Ultimately, our vision is to use tools and techniques from functional programming to build a new generation of tools that can enable non-expert end users to effectively work with desktop manufacturing devices. This paper presents a first step in that direction by laying the programming languages foundation necessary to approach the problem in a rigorous and principled way.

The rest of the paper is organized as follows: Section 2 presents a brief overview of 3D printing, which is one of the most popular desktop class manufacturing techniques and the primary motivation behind our synthesis tool. Section 3 describes a model of CAD programming as a purely functional programming language equipped with a denotation to 3D solids. It also presents a formalism of triangular polygon meshes along with a denotation to 3D solids. Section 4 presents a meaning-preserving compilation algorithm from CAD to STL and a proof sketch for compiler correctness. Section 5 presents our synthesis algorithm for reverse compiling STL to CAD, along with a definition and proof of correctness which introduces the technique of "geometric oracles" to reason about the algorithm. Section 6 presents a set of case studies demonstrating the feasibility of these ideas by synthesizing editable CAD from unstructured STL objects selected from Thingiverse Section 8 describes key areas for future work. Section 9 concludes.

${ }^{1}$ Our tool is available here: http://incarnate.uwplse.org/ 


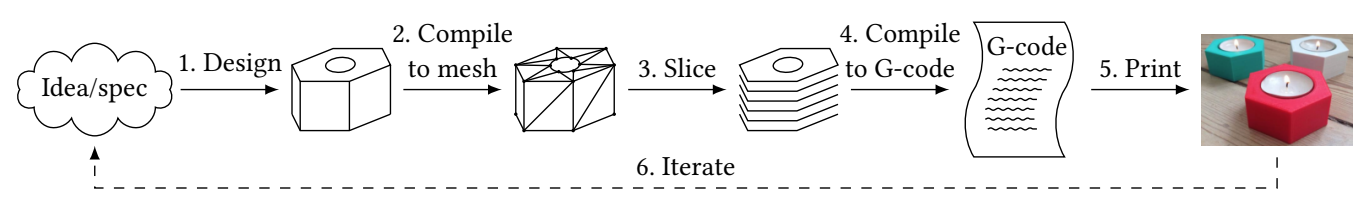

Fig. 1. 3D printing workflow depicting the 6 steps described in Section 2.1.

\section{3D PRINTING BACKGROUND AND MOTIVATION}

Among various desktop-class manufacturing devices, 3D printers are the most widely adopted due partly to the fact that they are relatively safer to use compared to desktop versions of other devices such as laser cutters and CNC mills. Figure 1 shows a 3D printing workflow, which typically comprises six steps: (1) design, (2) compile to mesh, (3) slice, (4) compile to G-code, (5) print, and (6) iterate.

\subsection{D printing as Compilation}

Just as programmers rarely write assembly directly, users of 3D printers do not write direct instructions for the motors. They instead produce them via compilation from a high-level design based on a specification or idea, created in a computer-aided design (CAD) software such as OpenSCAD [OpenSCAD 2018], Rhino [Rhinoceros 2018], or SketchUp [SketchUp 2018]. This compilation process is complex and, similar to classical compilers, typically proceeds through a sequence of intermediate languages that we now describe.

After designing a model, the next step is to compile it to a surface mesh representation. A surface mesh is a triangulation of the surface of the $3 \mathrm{D}$ object represented by the design. The third step slices the mesh into 2D layers that are stacked on top of each other during the printing phase. The next step generates $G$-code, which is similar to assembly-level instructions for manufacturing devices [Smid 2003]. The G-code is then interpreted by printer firmware to control the print (much as Postscript can be sent directly to many $2 \mathrm{D}$ printers).

In most desktop-class 3D printers, a spool feeds filament (typically a plastic) into an extruder, which heats and melts the filament before extruding it through a nozzle onto a print bed. It is this extruder that is controlled by the G-code, via low-level commands that actuate stepper motors to move the print head in any of three dimensions.

After completing the printing step, the user compares the output with the original specification and decides to either iterate over the above steps or terminate the process. Designing a CAD model is analogous to writing a program in a high level programming language. Converting it to a 3D mesh is similar to an intermediate representation. Slicing it and generating G-code is analogous to generating assembly. Viewing this workflow as a compiler has multiple advantages. It brings the formalisms of programming languages theory to bear, which in turn helps in reasoning about the correctness of the pipeline. It also supports the implementation of additional tools that can make these systems more accessible to end users (see Section 2.2).

\subsection{Synthesis Example}

Consider the model of a hexagonal candle holder from Thingiverse [Thingiverse 2018a] shown in Figure 2a. Like most models shared in online repositories, it is shared as a mesh. (Figure $2 \mathrm{~b}$ shows the rendering of the mesh.) Figure $2 \mathrm{c}$ shows a very small snippet from the mesh showing just one face and its normal direction vector. The full mesh is made of 548 triangular faces and is about 4000 lines long in the STL format. This vast sea of triangle vertices does not explicitly convey structural information about the object's shape. 


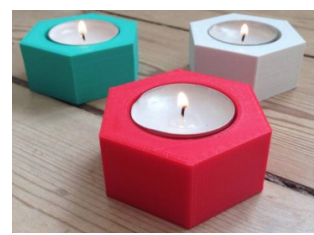

(a) Candle holder.

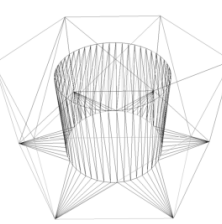

(b) Rendered mesh.

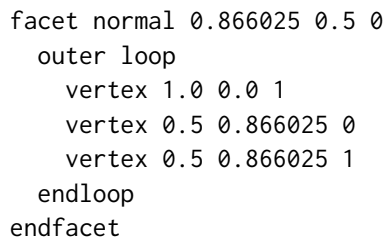

(c) Snippet from the STL mesh.

Fig. 2. Candle holder from Thingiverse [Thingiverse 2018a] after printing, a 3D rendering of its STL mesh, and a snippet from the STL mesh showing one triangular face. Each face is represented by three vertices and a normal vector that points outward from the $3 \mathrm{D}$ object.

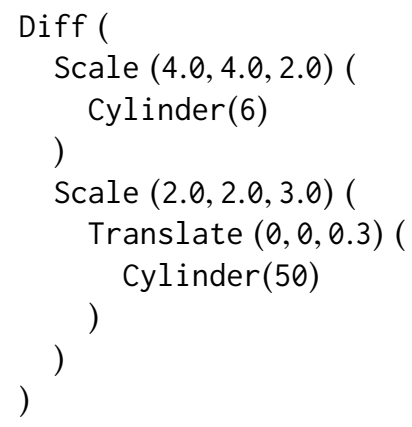

(a) $\lambda \mathrm{CAD}$ program for candle holder.

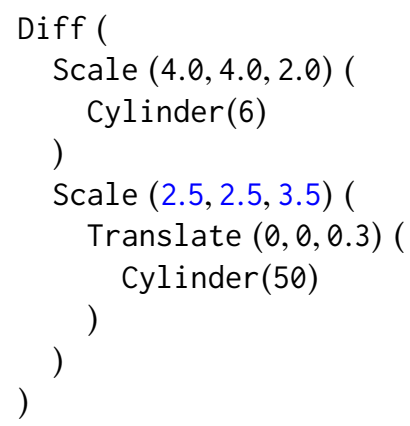

(b) Edited $\lambda \mathrm{CAD}$ program in blue.

Fig. 3. $\lambda \mathrm{CAD}$ program for the hexagonal candle holder in Figure 2, and example of a modification that changes the dimension of the hole (in blue). The hexagonal outer part is represented by Cylinder ( 6 ) (a cylindrical prism with 6 sides), and the hole is represented by Cylinder(50) (approximation of a cylinder using 50 sides). As shown, in $\lambda$ CAD this edit is done by changing the scaling factor for the cylindrical hole.

A user may want to make different modifications to the model. For example, they may wish to (1) change the depth/width of the candle hole, (2) tilt the hole (to make a holder for other items), (3) change the shape of the hole from a cylinder to a star-like prism or a cuboid, or (4) make a larger holder for two candles by combining two copies of the object. Making these edits to the mesh is non-trivial because the user must maintain certain geometric well-formedness constraints in order to ensure that the model is printable. On the other hand, having access to a higher-level representation of the model that contains more structural information such as any $\mathrm{CAD}$ representation would make these tasks quite easy. Figure $3 \mathrm{a}$ shows the code for this model in our language, $\lambda \mathrm{CAD}$. It shows that to make this model, one can create a 6-sided prism primitive (Cylinder (6)) and subtract a (high-degree approximation of a) cylindrical hole (Cylinder (50)) from its center. With access to this program, editing it is straightforward. Figure $3 \mathrm{~b}$ shows a modification to the model that changes the dimensions of the hole; an example of a modification requested by a user on the Thingiverse website [Thingiverse 2018a].

To summarize, (1) designing 3D models in CAD from scratch is difficult but editing an existing CAD program is relatively easy, and, (2) sharing models in a standardized mesh format makes them more accessible to users but editing them is difficult and even impossible in some cases. Based on these two observations, we came up with an alternate strategy that has the best of both worlds: We 


$\begin{aligned} C::= & \text { Mesh } M \\ \mid & \text { Empty } \\ & \text { Cube } \\ & \text { Cylinder } \mathbb{N} \\ & \\ & \text { Affine } \mathbb{R}^{3 \times 3} \mathbb{R}^{3} C \\ \mid & \text { Binop op } C C\end{aligned}$

$$
\begin{aligned}
& \llbracket \text { Mesh } m \rrbracket=\llbracket m \rrbracket \\
& \llbracket \text { Empty } \rrbracket=\{\} \\
& \llbracket \text { Cube } \rrbracket=(0,1)^{3} \\
& \llbracket \text { Affine } p q c \rrbracket=\{p v+q \mid v \in \llbracket c \rrbracket\} \\
& \llbracket \text { Binop o } c_{1} c_{2} \rrbracket=\llbracket c_{1} \rrbracket \llbracket o \rrbracket \llbracket c_{2} \rrbracket \\
& \llbracket \text { Union } \rrbracket=\cup \quad \llbracket \text { Inter } \rrbracket=\cap \quad \llbracket \text { Diff } \rrbracket=\backslash
\end{aligned}
$$

Fig. 4. CAD syntax and semantics. CAD programs denote to regular open sets in $\mathbb{R}^{3}$. Affine transformations are given by an invertible $3 \times 3$ matrix and translation vector. Binary operators denote to set operations. Mesh denotation is detailed below.

describe the first synthesis algorithm (Section 5) that automatically finds a CAD program from a surface mesh. This approach gives the users a high level CAD program to get started with, prevents them from having to make tedious mesh modifications while still allowing them to download mesh models from the internet.

\section{FORMALIZING CAD AND MESH}

CAD and mesh can be viewed as two fundamentally different ways of representing an object in 3D space. While CAD representations are based on solid geometry, mesh representations are based on surface geometry. Any translation between these two conceptually different approaches requires finding a way to map the concepts from one to the other. To that end, this section presents the syntax and denotational semantics for two languages for 3D modeling, $\lambda \mathrm{CAD}$, a high-level functional programming language, and Mesh, an intermediate surface representation based on industry-standard formats.

\section{1 $\lambda$ CAD Language}

We designed and implemented $\lambda \mathrm{CAD}$, a functional programming language with primitives for representing and manipulating geometric objects. Since the other features are standard, this section focuses on the syntax and semantics of the geometric fragment of the language.

Figure 4 shows the syntax of the geometric core of $\lambda C A D$. It supports (1) $3 D$ primitives such as Cube and Cylinder, (2) affine transformations such as translation (Translate), rotation about $\mathrm{X}, \mathrm{Y}$, and $\mathrm{Z}$ axes (RotateX, RotateY, RotateZ), uniform and non-uniform scaling (Scale), and, (3) set-theoretic operations: Union, Difference, and Intersection. The primitives represent 3D shapes with unit measures. For example, Cube has all sides of unit length and the bottom left corner at the origin, $(0,0,0)$. Cylinder $(n)$ is an $n$-sided prism with unit radius and height whose base is centered at the origin. Note that, as presented, all primitive objects are piecewise linear, thus requiring curves to be approximated. Truly curved primitives are interesting and possible, but complicate the semantics, compilation, and synthesis approaches discussed in this paper. The possibility of developing a compositional notion of equality between piecewise-linear approximations to curves in a way that supports correctness proofs for compilation and synthesis is a significant challenge left for future work. In this paper, we represent curves using approximations-for example, to represent a cylinder we set $n=50$ in $C y$ linder $(n)$. All the affine transformations are represented using an invertible $3 \times 3$ matrix and a $3 \mathrm{D}$ vector in the core CAD syntax in Figure 4 . $\lambda \mathrm{CAD}$ also supports user-provided raw meshes using the Mesh construct.

Figure 4 describes a denotational semantics for CAD that maps each object to the set of 3D points inside it. The primitive Empty maps to the empty set, while Cube maps to the set of all points whose 


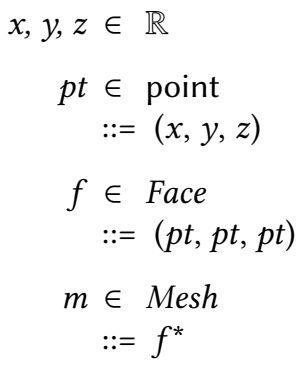

(a) Syntax of Mesh.

$$
\begin{array}{rcl}
r & \in & \mathbb{R} \\
d & \in & \text { Direction } \\
d & := & (r, r, r) \\
h & \in & \text { HalfLine } \\
h & := & (p t, d) \\
\text { Midpoint } & : & \text { Face } \rightarrow \text { point } \\
\text { Norm } & : & \text { Mesh } \times \text { Face } \rightarrow\{L, R\} \\
\text { On } & : & \text { Mesh } \times \text { Face } \rightarrow \text { bool }
\end{array}
$$

(b) Mesh functions used in the compiler (Figure 9).

Fig. 5. Syntax and auxiliary definitions for mesh. In Figure 5b, Midpoint is the centroid of a face. A point is On a face if it is coplanar with the face, and is in the interior of the face or on one of its edges.

$x, y$, and $z$ values lie in $(0,1)$. Other primitives are similarly straightforward. Affine transformations are denoted by applying the transformation to every point in the denotation of $e$. The denotation of Union $\left(e_{1}, e_{2}\right)$ is the union of the denotations of $e_{1}$ and $e_{2}$. Intersection and difference are similar.

\subsection{Surface Mesh}

A surface polygon mesh is a geometric representation of the surface of an object in 3D space using vertices, edges, and faces. The faces of a mesh are typically convex polygons. In this paper, we formalize triangular meshes as shown in Figure 5a. A mesh is a list of faces, each of which is a triangle represented by its three vertices. This simple and flat representation is as expressive as other representations [Grimm 2004] yet serves as a high-level executable specification, which could be used, for example, to differentially test against more sophisticated implementations such as STL and OFF [Grimm 2004; OFF 2018]. Section 3.2.2 describes how we use normals to determine which side of a triangular face is inside/outside a $3 \mathrm{D}$ object.

3.2.1 Valid Mesh. In order for a 3D CAD model to be printable, the mesh should be valid. Invalid meshes can have a variety of problems such as zero volume, holes, and dangling faces, which make them unfit for printing. A valid 3D mesh is one that satisfies the following invariants:

- no overlapping or intersecting faces

- no edges that occur in an odd number of faces

- not open, i.e. should not have holes (or missing faces). This happens if an edge is on an odd number of faces.

Figure 6 shows 2D analogues of some invalid meshes. We use 2D in the figure for simpler visualization. In 2D, the faces are segments instead of triangular planes. The analogue of edges in 2D are the vertices. The first figure in Figure 6 shows a 2D mesh that is open. This can happen when a vertex appears in an odd number of segments. The second figure is another example of an invalid mesh with a lone face. The $3 \mathrm{D}$ analogue of this is a mesh with an extra triangular face. The third figure is an example of a mesh with zero area. An example of this in 3D would be a mesh with just one triangular face, which would have zero volume.

3.2.2 Sides of a Mesh. Knowing the vertices of the faces of a mesh is not sufficient to determine the "inside" and "outside" of the shape. This information is given by normal vectors for each face of a mesh, which are unit vectors orthogonal to the face that point toward the outside of the shape. We use $L$ (left) and $R$ (right) to indicate the two possible directions for normals (Figure 5b), depending on whether the left-hand or right-hand rule should be used on the given face. Typical industrial 


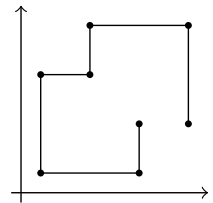

(a) 2D model with missing face.

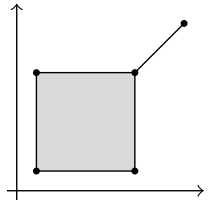

(b) 2D model with extra face.

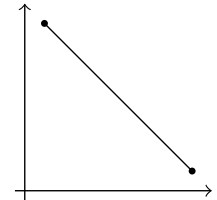

(c) Flat 2D model with zero area

Fig. 6. Analogues of ill-formed meshes in 2D (used for simpler visualization). A 2D face is a line segment whereas a 3D face is a triangular plane. Thus, a missing face in 3D would be a missing triangle, an extra face would be an extra triangle, and a mesh with zero volume would be a plane.

formats store normal vectors in the representation of each face [Grimm 2004], but for conceptual parsimony, we instead compute normals as required using global properties of the mesh.

Specifically, in a valid mesh, the normal vectors can be computed once we have a way of determining whether a point is inside or outside the mesh. For this, we use the well-known method of casting rays [de Berg 1997]. A ray, or halfline, $h$ is represented by a starting point, $p t$ and a direction, $d$, as shown in Figure 5b. A point is inside a 3D mesh if there is a good halfline starting at the point that crosses an odd number of faces of the mesh (Figure 7). A good halfline is one that does not intersect the mesh at its vertices or edges. An important result is that many good halflines exist for any point not on the boundary of the mesh (Theorem 1).

Theorem 1. For any valid mesh $m$ and point $p t$ not on the boundary of $m$, almost all directions $d$ result in good halflines (that is, all directions outside a set of measure 0 result in good halflines).

Proof. The edges and vertices of $m$, when projected onto a unit sphere around $p t$, form a set of measure 0 . Any direction $d$ on the sphere outside of this set forms a good halfline for $p t$.

It is also essential that the choice of halfline does not matter in a valid mesh (Theorem 2).

Theorem 2. For any valid mesh $m$, point $p t$ not on any face of $m$, and good halflines $h_{1}$ and $h_{2}$ each starting at $p t$, the halfline $h_{1}$ intersects $m$ an odd number of times if and only if $h_{2}$ intersects $m$ an odd number of times.

Proof. First, note that there exists a plane containing $h_{1}$ and $h_{2}$. The intersection of this plane and the mesh is a simple 2D polygon $m_{2}$ (the mesh is valid so faces do not intersect) containing $p t$ (since both $h_{1}$ and $h_{2}$ contain $p t$ ). We must show that $h_{1}$ and $h_{2}$ intersect $m_{2}$ with equal parity. As shown by, for example, Hormann and Agathos [Hormann and Agathos 2001], this parity is equal to a formula over the angles between $m_{2}$ 's edges, and must thus be the same for $h_{1}$ and $h_{2}$.

We can now compute normals for a face using any test point $p t$ in the interior of $f$ (we use Midpoint $(f)$ in our implementation). Consider any good halfline $h$ for $p t$. If $h$ crosses $m$ an odd number of times, then $h$ lies on the same side of $f$ as the outward-facing normal. Otherwise, it is on the opposite side.

This technique also determines a denotational semantics for meshes that denotes a mesh to the set of points inside it, thus enabling easy comparison with the denotation of CAD objects. Figure 7 defines this semantics based on face and halfline intersection. The intersection of a face and a halfline can have three outcomes: (1) None indicates that the face and the halfline do not intersect at any point, (2) InteriorPt indicates that the halfline goes through the face at exactly one point in its interior, and (3) Other indicates all other possible interactions of a face and a halfline: they are coplanar and the halfline goes through an edge or a vertex of the face. InsideVia (m, pt, d) is a predicate that defines when $\mathrm{pt}$ is inside the mesh $\mathrm{m}$ : if there exists a direction $\mathrm{d}$, such that for 


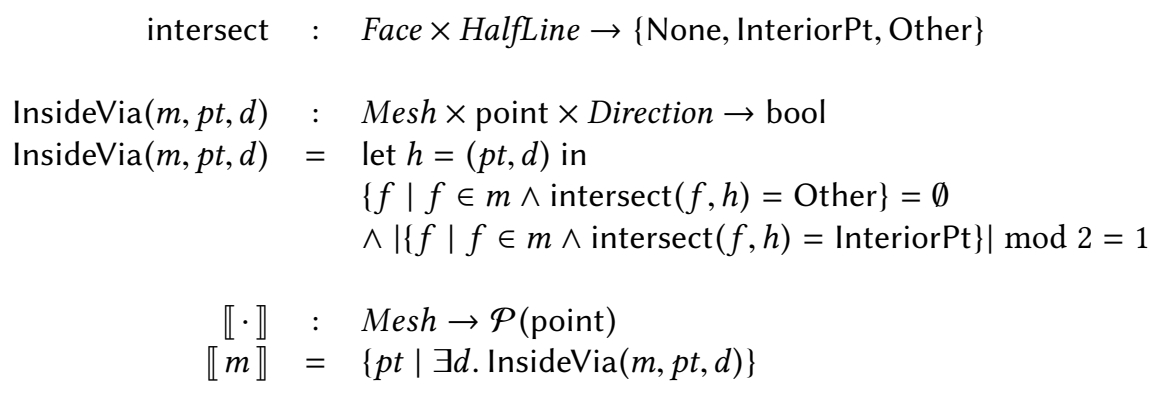

Fig. 7. Semantics of Mesh using intersection of faces with halflines (rays).

the halfline $h=(p t, d),(1)$ there is no face in $m$ that results in an Other intersection with $h$, and (2) $\mathrm{h}$ crosses the mesh at an odd number of faces, then $\mathrm{pt}$ is inside the mesh, $\mathrm{m}$.

\section{3D PRINTING AS COMPILATION}

This section presents a meaning-preserving compiler that generates a triangular mesh from $\lambda \mathrm{CAD}$. The compiler's specification is given in terms of the geometric denotational semantics of the source and target languages. The straightforward compilation algorithm described here is used in some form or another in all industrial CAD tools. Our contributions are (1) to formalize it in terms of structural recursion and denotational semantics, which enables (2) a proof of correctness.

Figure 9 defines the compiler as a recursive function on the syntax of the CAD program. The output of compiling a Mesh $m$ construct is the underlying mesh, $m$. Compiling an Empty CAD model simply generates an empty mesh. The mesh for Cube is as defined in Figure 9. Since we use pre-defined meshes to approximate curves in this paper, the output of compiling them is simply the corresponding pre-defined mesh. For affine transformations, the compiler generates the mesh by applying the transformation to the result of the recursive call.

\subsection{Compiling CSG Operations and Mesh Splitting}

To translate the set-theoretic binary operations, the compiler uses corresponding functions on meshes, mBop(Union) $\left(m_{1}, m_{2}\right), m B o p$ (Difference) $\left(m_{1}, m_{2}\right)$, and $m B o p$ (Intersection) $\left(m_{1}, m_{2}\right)$, shown in Figure 9. These operations are non-trivial for overlapping meshes, since the faces of the resulting mesh are a complex subset of a refinement of both input meshes. If two input faces overlap, then some parts of each face may be discarded in the output, while other parts remain. Care is required to preserve the mesh invariants defined in Section 3.2.1.

The set-theoretic mesh operations first split the input meshes, defined by the relation split (used in Figure 9) that takes two potentially intersecting meshes and returns two equivalent meshes that intersection only at vertices and edges. The operation split preserves the semantics of the meshes, as proved in Theorem 4 . The functions $\operatorname{mBop}($ Union $)\left(m_{1}, m_{2}\right), \operatorname{mBop}($ Difference $)\left(m_{1}, m_{2}\right)$, and $m B o p$ (Intersection $)\left(m_{1}, m_{2}\right)$ then determine which faces from the split meshes $m_{1}^{\prime}$ and $m_{2}^{\prime}$ should be kept in the final mesh and which ones should be discarded.

$m B o p($ Union $)\left(m_{1}^{\prime}, m_{2}^{\prime}\right)$ keeps faces from $m_{1}^{\prime}$ that are outside $m_{2}^{\prime}$ as well as faces from $m_{2}^{\prime}$ that are outside $m_{1}^{\prime}$. For faces from $m_{1}^{\prime}$ that are also on $m_{2}^{\prime}$ (on is defined in Figure $5 \mathrm{~b}$ ), if the face has the same normal, then it is kept, otherwise it is discarded. This is illustrated (in 2D) in Figure 8. $m B o p($ Difference $)\left(m_{1}^{\prime}, m_{2}^{\prime}\right)$ keeps faces from $m_{1}^{\prime}$ that are outside $m_{2}^{\prime}$, faces from $m_{2}^{\prime}$ that are inside $m_{1}^{\prime}$. For faces from $m_{1}^{\prime}$ that are also on $m_{2}^{\prime}$, if the face has the same normal $(L$ or $R)$, then it is discarded, otherwise it is kept. $m B o p($ Intersection $)\left(m_{1}^{\prime}, m_{2}^{\prime}\right)$ keeps faces from $m_{1}^{\prime}$ that are inside $m_{2}^{\prime}$, faces from 

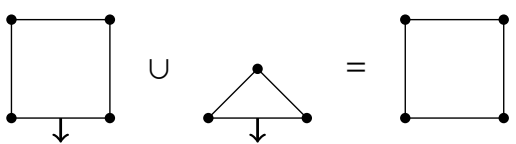

(a) Keeping a common edge during mesh union.

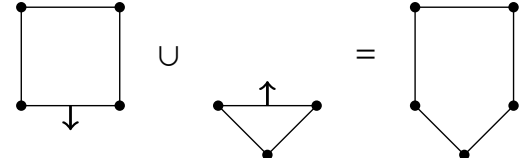

(b) Removing a common edge during mesh union.

Fig. 8. Examples (in 2D) demonstrating when common faces are retained or removed by mBop(Union).

$m_{2}^{\prime}$ that are inside $m_{1}^{\prime}$. For faces from $m_{1}^{\prime}$ that are also on $m_{2}^{\prime}$, if the face has the same normal ( $L$ or $R$ ), then it is kept, otherwise it is discarded.

Definition 3. If $f_{i}$ is a face on $m_{1}$ that overlaps with some face on $m_{2}$, split splits $f_{i}$ in to $f_{i 1}, f_{i 2}, \ldots, f_{\text {in }}$ such that

$$
\bigcup_{j=1}^{n} f_{i j}=f_{i} \text { and } \bigcap_{j=1}^{n} f_{i j}=\emptyset .
$$

Theorem 4 (Mesh splitting correctness). Given two valid meshes, $m_{1}$ and $m_{2}$, split $\left(m_{1}, m_{2}, m_{1}^{\prime}, m_{2}^{\prime}\right)$ is a relation such that:

$$
\llbracket m_{1} \rrbracket=\llbracket m_{1}^{\prime} \rrbracket \text { and } \llbracket m_{2} \rrbracket=\llbracket m_{2}^{\prime} \rrbracket
$$

Proof. We prove $\llbracket m_{1} \rrbracket=\llbracket m_{1}^{\prime} \rrbracket$; the proof for the second part is similar. Let $p t$ be an arbitrary point. We show $p t \in \llbracket m_{1} \rrbracket \Longleftrightarrow p t \in \llbracket m_{1}^{\prime} \rrbracket$. Consider any halfline $h$ that is good for $p t$ and $m_{1}^{\prime}$ (such a halfline exists by Theorem 1), and consider the points of intersection between $h$ and the two meshes $m_{1}$ and $m_{1}^{\prime}$. By definition, split ensures that the intersection of split faces are disjoint, so each point of intersection between $h$ and $m_{1}$ lies on exactly one face of $m_{1}^{\prime}$. Conversely, split also ensures that the union of split faces give the original face, so each intersection point between $h$ and $m_{1}^{\prime}$ also lies on a face of $m_{1}$. Thus the intersection points along $h$ are exactly the same for $m_{1}$ and $m_{1}^{\prime}$.

\subsection{Compiler Correctness}

Our definition of compiler correctness is based on the denotational semantics we described in Section 3. Specifically, we prove that the compiler returns a mesh with the same denotation as the input CAD program. For binary operations, we provide the proof for union. The cases for intersection and difference are similar and hence omitted.

Theorem 5 (Compiler correctness). For all CAD expressions $e, \llbracket \operatorname{compile}(e) \rrbracket=\llbracket e \rrbracket$.

Proof. By induction on $e$. We show a few representative cases.

Case Empty:

$$
\begin{aligned}
\llbracket \text { compile }(\text { Empty }) \rrbracket & =\llbracket[] \rrbracket \\
& =\emptyset \\
& =\llbracket \text { Empty }
\end{aligned}
$$

By definition of compile(). By definition of mesh $\llbracket \rrbracket$. By definition of CAD $\llbracket$.

Case Cube:

$$
\begin{aligned}
\llbracket \text { compile }(\text { Cube }) \rrbracket & =\llbracket m_{\text {cube }} \rrbracket & & \text { By definition of compile }() . \\
& =\llbracket \text { Cube } & & \text { By Lemma } 6 .
\end{aligned}
$$




$$
\begin{aligned}
& \operatorname{compile}(\text { Mesh } m)=m, \operatorname{compile}(\text { Empty })=[], \operatorname{compile}(\text { Cube })=m_{\text {cube }} \\
& C_{2}=[((0,0),(1,0),(1,1)),((0,0),(0,1),(1,1))] \\
& m_{\text {cube }}=\left[f\left(j, C_{2}\right) \mid f \in\left[i_{x}, i_{y}, i_{z}\right], j \in[0,1]\right] \\
& i_{x}, i_{y}, i_{z} \quad: \quad \mathbb{R}^{2} \rightarrow \mathbb{R}^{3} \\
& i_{x}\left(x_{0},(a, b)\right)=\left(x_{0}, a, b\right) \\
& i_{y}\left(y_{0},(a, b)\right)=\left(a, y_{0}, b\right) \\
& i_{z}\left(z_{0},(a, b)\right)=\left(a, b, z_{0}\right) \\
& \text { compile }(\text { Affine p } q c)=\operatorname{map}_{\text {vertex }}(\lambda v \cdot p v+q)(\text { compile }(c)) \\
& \text { compile }\left(\text { Binop o } c_{1} c_{2}\right)=\operatorname{mBop}(o)\left(\operatorname{compile}\left(c_{1}\right), \text { compile }\left(c_{2}\right)\right) \\
& \text { mBop(Union) }\left(m_{1}, m_{2}\right)=\text { let } m_{1}^{\prime}, m_{2}^{\prime} \text { s.t., split }\left(m_{1}, m_{2}, m_{1}^{\prime}, m_{2}^{\prime}\right) \text { in } \\
& {\left[f \in m_{1}^{\prime} \mid \nexists d \text {. InsideVia }\left(m_{2}^{\prime}, \operatorname{Midpoint}(f), d\right)\right]++} \\
& {\left[f \in m_{2}^{\prime} \mid \nexists d \text {. InsideVia }\left(m_{1}^{\prime}, \operatorname{Midpoint}(f), d\right)\right]++} \\
& {\left[f \in m_{1}^{\prime} \mid \operatorname{On}\left(m_{2}^{\prime}, f\right) \wedge \operatorname{Norm}\left(m_{1}^{\prime}, f\right)=\operatorname{Norm}\left(m_{2}^{\prime}, f\right)\right]} \\
& m B o p(\text { Difference })\left(m_{1}, m_{2}\right)=\text { let } m_{1}^{\prime}, m_{2}^{\prime} \text { s.t., split }\left(m_{1}, m_{2}, m_{1}^{\prime}, m_{2}^{\prime}\right) \text { in } \\
& {\left[f \in m_{1}^{\prime} \mid \nexists d \text {. InsideVia }\left(m_{2}^{\prime}, \operatorname{Midpoint}(f), d\right)\right]++} \\
& {\left[f \in m_{2}^{\prime} \mid \exists d \text {. InsideVia }\left(m_{1}^{\prime}, \operatorname{Midpoint}(f), d\right)\right]++} \\
& {\left[f \in m_{1}^{\prime} \mid \operatorname{On}\left(m_{2}^{\prime}, f\right) \wedge \operatorname{Norm}\left(m_{1}^{\prime}, f\right) \neq \operatorname{Norm}\left(m_{2}^{\prime}, f\right)\right]} \\
& \text { mBop(Intersection })\left(m_{1}, m_{2}\right)=\text { let } m_{1}^{\prime}, m_{2}^{\prime} \text { s.t., split }\left(m_{1}, m_{2}, m_{1}^{\prime}, m_{2}^{\prime}\right) \text { in } \\
& {\left[f \in m_{1}^{\prime} \mid \exists d \text {. InsideVia }\left(m_{2}^{\prime} \text {, Midpoint }(f), d\right)\right]++} \\
& {\left[f \in m_{2}^{\prime} \mid \exists d \text {. InsideVia }\left(m_{1}^{\prime}, \operatorname{Midpoint}(f), d\right)\right]++} \\
& {\left[f \in m_{1}^{\prime} \mid \operatorname{On}\left(m_{2}^{\prime}, f\right) \wedge \operatorname{Norm}\left(m_{1}^{\prime}, f\right)=\operatorname{Norm}\left(m_{2}^{\prime}, f\right)\right]}
\end{aligned}
$$

Fig. 9. Representative cases of CAD-to-Mesh compiler. Midpoint, On and Norm are as defined in Figure 5b.

Case Affine $p q e^{\prime}$ : Let $m^{\prime}$ represent compile $\left(e^{\prime}\right)$.

$\llbracket$ compile $\left(\right.$ Affine $\left.p q e^{\prime}\right) \rrbracket=\llbracket \operatorname{map}_{\text {vertex }}(\lambda v \cdot p v+q)\left(m^{\prime}\right) \rrbracket$

$$
\begin{aligned}
& =\left\{p v+q \mid v \in \llbracket m^{\prime} \rrbracket\right\} \\
& =\left\{p v+q \mid v \in \llbracket e^{\prime} \rrbracket\right\} \\
& =\llbracket \text { Affine } p q e^{\prime} \rrbracket
\end{aligned}
$$

By definition of compile().

By Lemma 7.

By induction hypothesis.

By definition of CAD $\llbracket$.

Case Union $e_{1} e_{2}$ : Let $m_{1}, m_{2}$ represent compile $\left(e_{1}\right)$ and compile $\left(e_{2}\right)$ respectively.

$$
\begin{aligned}
\left.\llbracket \text { compile(Binop Union } e_{1} e_{2}\right) \rrbracket & =\llbracket m B o p(\text { Union })\left(m_{1}, m_{2}\right) \rrbracket & & \text { By definition of compile }() . \\
& =\llbracket m_{1} \rrbracket \cup \llbracket m_{2} \rrbracket & & \text { By Lemma } 8 . \\
& =\llbracket e_{1} \rrbracket \cup \llbracket e_{2} \rrbracket & & \text { By induction hypothesis. } \\
& =\llbracket \text { Union } e_{1} e_{2} \rrbracket & & \text { By definition of CAD } \rrbracket .
\end{aligned}
$$

Lemma 6. $\llbracket m_{c u b e} \rrbracket=\llbracket C u b e \rrbracket$

Proof. $\quad(\subseteq)$ Suppose $p t \in \llbracket m_{\text {cube }} \rrbracket$. Then there exists $d$ such that InsideVia $(m, p t, d)$. Let $h$ be the half-line from $p t$ in direction $d$. Since $m_{c u b e}$ is convex, there is exactly one face through 


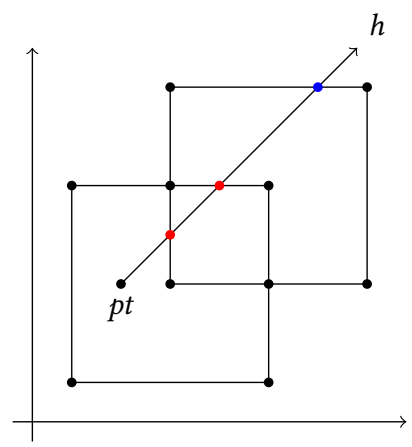

(a) Before

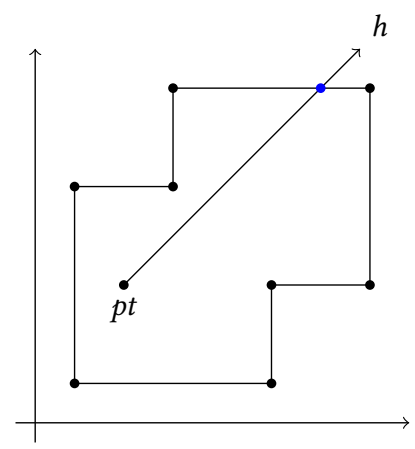

(b) After

Fig. 10. Examples demonstrating (in 2D) several cases in the proof of Lemma 8.

which $h$ passes. Let $f_{0}$ be this unique face and consider the intersection of $f_{0}$ and $h$. Since $h$ intersects $m_{\text {cube }}$ exactly once, $h$ must leave the cube at $f_{0}$. So just before leaving the cube at $f_{0}, h$ is inside the cube. Unless $p t \in C u b e, h$ would leave the cube again.

(つ) Suppose $p t \in \llbracket C u b e \rrbracket$. If $p t$ is on the boundary of Cube, say on face $f$, then let $d$ be the outward-facing normal of $f$, so that $h=(p t, d)$ intersects $m_{\text {cube }}$ exactly once. On the other hand, suppose $p t$ is in the interior of Cube. Then choose $d=(0,0,1)$, somewhat arbitrarily. Again, $h=(p t, d)$ intersects $m_{c u b e}$ exactly once.

Lemma 7. For all meshes $m$ and invertible affine transformations given by $p$ and $q$,

$$
\{p v+q \mid v \in \llbracket m \rrbracket\}=\llbracket \operatorname{map}_{\text {vertex }}(\lambda v \cdot p v+q) m \rrbracket .
$$

Proof. Let $p t$ be arbitrary.

$$
\begin{aligned}
p t \in\{p v+q \mid v \in \llbracket m \rrbracket\} & \Longleftrightarrow p^{-1}(p t-q) \in \llbracket m \rrbracket \\
& \Longleftrightarrow \exists d . \operatorname{InsideVia}\left(m, p^{-1}(p t-q), d\right) \\
& \Longleftrightarrow \exists d^{\prime} . \text { InsideVia }\left(\operatorname{map} \text { vertex }(\lambda v \cdot p v+q) m, p t, d^{\prime}\right) \\
& \Longleftrightarrow p t \in \llbracket \operatorname{map}_{\operatorname{vertex}}(\lambda v \cdot p v+q) m \rrbracket
\end{aligned}
$$

Lemma 8. For all meshes $m_{1}$ and $m_{2}$,

$$
\llbracket m B o p(\text { Union })\left(m_{1}, m_{2}\right) \rrbracket=\llbracket m_{1} \rrbracket \cup \llbracket m_{2} \rrbracket .
$$

Proof. Let $m_{3}=m B o p($ Union $)\left(m_{1}, m_{2}\right)$ and let $p t$ be an arbitrary point. We show $p t \in m_{3} \Longleftrightarrow$ $p t \in \llbracket m_{1} \rrbracket \vee p t \in \llbracket m_{2} \rrbracket$. Consider a ray $h$ that intersects only interior points of the faces of $m_{1}$ and $m_{2}$ (and thus also of $m_{3}$ ). It suffices to show that $h$ crosses an odd number of faces in $m_{3}$ iff it crosses an odd number of faces of $m_{1}$ or an odd number of faces of $m_{2}$.

Subdivide $h$ into $n$ contiguous regions $h_{i}$, separated by $h$ 's intersections with $m_{1}$ and $m_{2}$, which we call crossing points. The first region, $h_{0}$, starts at infinity and proceeds to the first crossing point. Each subsequent pair of regions is divided by a crossing point on the face of one or several of $m_{1}$, $m_{2}$, and $m_{3}$. These crossing points are not considered to be included in any regions. Finally, the region $h_{n-1}$ ends at $p t$, which is considered a part of that region, since it is not itself a crossing point. 
Since $m_{1}$ and $m_{2}$ are split, each $h_{i}$ is entirely inside or outside of $m_{1}$ and $m_{2}$. Since each face of $m_{3}$ is a face of either $m_{1}$ or $m_{2}$, each region $h_{i}$ is also entirely inside or outside of $m_{3}$. We now show that $h_{i}$ is inside $m_{3}$ iff it is inside $m_{1}$ or inside $m_{2}$. We proceed by induction on $i$ :

Case $i=0$ : The statement follows since $h_{0}$ is the infinitely long region, which lies outside all three meshes.

Case $i+1$ : Consider the crossing that happens between $h_{i}$ and $h_{i+1}$. There are 16 cases in total, depending on whether $h_{i}$ and $h_{i+1}$ lie inside or outside of $m_{1}$ and $m_{2}$. We illustrate four typical cases. A 2D example is described in Figure 10.

Case $h_{i}$ outside $m_{1}$ and $m_{2} ; h_{i+1}$ inside $m_{1}$ but outside $m_{2}$ : Consider the face $f$ that divides $h_{i}$ and $h_{i+1}$. The case hypothesis implies that $f$ is a face of $m_{1}$ but not $m_{2}$. This further means that $f$ is entirely outside of $m_{2}$, and so $f$ is also a face of $m_{3}$ by definition of $m B o p$ (Union). Thus $h$ also crosses $m_{3}$ at $f$, and so $h_{i+1}$ is inside $m_{3}$.

Case $h_{i}$ inside $m_{1}$ but outside $m_{2} ; h_{i+1}$ inside both $m_{1}$ and $m_{2}$ : The crossing face $f$ is a face of $m_{2}$, which is entirely inside $m_{1}$, and thus not included in $m_{3}$. Inductively, $h_{i}$ is inside $m_{3}$, and since $f$ is not in $m_{3}, h_{i+1}$ is also inside $m_{3}$.

Case $h_{i}$ inside both $m_{1}$ and $m_{2} ; h_{i+1}$ outside both $m_{1}$ and $m_{2}$ : The crossing face $f$ is a face of both $m_{1}$ and $m_{2}$, and $f$ 's normals with respect to each mesh point in the same direction. Further, these normals are on the same side of $f$ as $p t$. Thus $f$ is a face of $m_{3}$. Inductively, $h_{i}$ is in $m_{3}$, and so it crosses out of $m_{3}$ for $h_{i+1}$.

Case $h_{i}$ inside $m_{1}$ but outside $m_{2} ; h_{i+1}$ inside $m_{2}$ but outside $m_{1}$ : The crossing face $f$ is a face of both $m_{1}$ and $m_{2}$, but the normals point in opposite directions. In $m_{1}$, the outward normal is on the same side as $p t$, while for $m_{2}$ it is on the opposite side. Thus, no copy of the face appears in $m_{3}$. Inductively, $h_{i}$ is in $m_{3}$, and since the crossing face is not in $m_{3}, h_{i+1}$ is as well.

The remaining cases are similar.

\subsection{Implementation and Challenges}

Implementing the $\mathrm{CAD}$ compiler required several nontrivial computational geometry routines, which involved issues from 3D geometry as well as numerical computing. This section describes some design decisions targeted at reducing the burden of implementing the compiler.

4.3.1 $1 D \rightarrow 3 D$. Problems that arise in $3 \mathrm{D}$ geometry often have analogous problems in lower dimension. Understanding which parts of the problem cut across all dimensions versus those that arise only in 3D helped us develop clean solutions that are as dimension-agnostic as possible. To that end, we first implemented a $1 \mathrm{D}$ CAD compiler, then moved on to $2 \mathrm{D}$ and finally to $3 \mathrm{D}$. $\lambda \mathrm{CAD}$ supports all three dimensions. An example of a dimension agnostic concept in our compiler is the technique for compiling CSG operations in Section 4.1. On the other hand, the technique for finding the intersection of a face and a halfline in Section 3.2.2 is a geometric operation, which is more complex in 3D where faces are triangular planes and halflines are 3D rays than in 2D where faces are $2 \mathrm{D}$ segments and halflines are $2 \mathrm{D}$ rays.

1-dimensional CAD. : 1D CAD objects are simply line segments represented by $1 \mathrm{D}$ end points. The only affine transformations in $1 \mathrm{D}$ are translation and scaling. The binary set-theoretic operations are analogous in all dimensions. A 1D CAD compiler compiles a 1D CAD program to generate a 1D mesh. Figure 11 shows a 1D CAD program and the corresponding mesh. Segment represents a unit segment starting at 0 and ending at 1 . A face of a $1 \mathrm{D}$ mesh is merely a $1 \mathrm{D}$ point. As explained in section 3.2, a valid 1D mesh should not have repeating faces or odd number of faces. 
1D

Translate (5)

(Scale (7) Segment)
$2 \mathrm{D}$

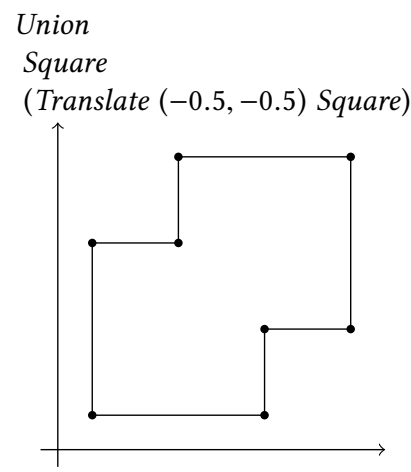

3D

Union

Cube

(Translate $(0,0.5,0.5)$ Cube $)$

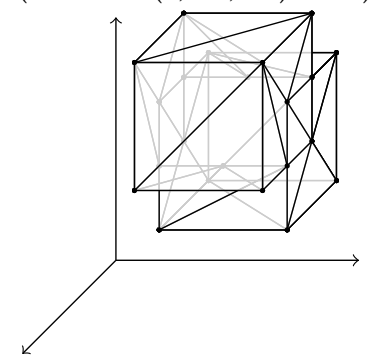

Fig. 11. Examples of 1D, 2D, 3D CADs and meshes. In order to keep the figures simple, the axes are not shown to intersect at the origin.

2-dimensional CAD. : 2D CAD objects include rectangles, squares, circles, triangles etc. In 2D, affine transformations include those from 1D (i.e. translation and scaling, but with 2D vectors) together with rotations about the origin. A 2D mesh consists of faces that are line segments ending in vertices. Figure 11 shows a $2 \mathrm{D}$ CAD program and the mesh generated by our compiler.

3-dimensional CAD. : In 3D, rotations about many different axes are possible. In our implementation, we provide convenient syntax for rotating about the coordinate axes, rotateX, rotateY, rotateZ. Translation and scaling are obviously possible, but using 3D vectors. Figure 11 shows a 3D CAD program and the corresponding triangular mesh.

4.3.2 Fully Functorial Design. We designed our compiler infrastructure in a hierarchical manner using a fully functorial approach which allows us to swap out components of the compiler with other implementations. OCaml's module system facilitated this design decision. This is particularly useful for differential testing our compiler against other solid geometry based tools such as OpenSCAD [OpenSCAD 2018], swapping our geometry module with another computational geometry library for comparison, and in tackling numerical issues. The geometric functionalities in our compiler and synthesis implementation are conceptually designed to execute using real numbers. Since reals are only approximated by floating point numbers, running these routines using floating point often leads to rounding errors due to semantic mismatch between floats and reals, and undecidable branching. We implemented several number systems with varying levels of accuracy and were able to use them interchangeably as and when required. All modules are functorized over a number system, whose signature contains basic arithmetic, square root, and trigonometric operations. We have implemented this signature using floating points, arbitrary precision number systems such as MPFR [Fousse et al. 2007; Zimmermann 2010], and exact arithmetic (see Section 8.1).

\section{SYNTHESIZING EDITABLE CAD BY REVERSE COMPILATION}

To demonstrate how the foundations established in previous sections can help develop better tools for desktop-manufacturing users, we describe a novel algorithm for "reverse compiling" meshes to $\mathrm{CAD}$ programs that recaptures the high-level structure of a design. In this section we show how our $\mathrm{CAD}$ and mesh formalizations suggest a natural search strategy for synthesis. In Section 6 we detail case studies that demonstrate the promise of this approach: once meshes are reverse 

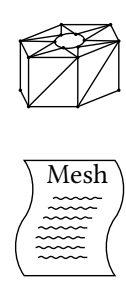

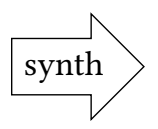

$$
\begin{aligned}
& \text { Diff }( \\
& \text { Scale }(2,2,2)( \\
& \text { Cylinder(6) } \\
& \text { Cylinder(50) }
\end{aligned}
$$
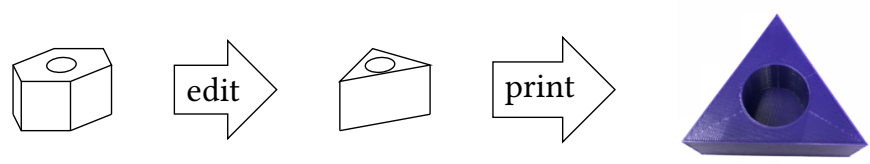

Fig. 12. Synthesis workflow: starting with a mesh for a 3D model, our synthesis tool can reverse engineer a CAD program, which can be easily edited to get a different 3D model.

$$
\begin{aligned}
& \left.E::=[\cdot] \mid \text { Affine } \mathbb{R}^{3 \times 3} \mathbb{R}^{3} E \mid \text { Binop op } E C \mid \text { Binop op (Mesh } \mathrm{m}\right) E \\
& S::=A \mid \text { Binop (Union } \mid \text { Diff) } S S \quad A::=P \mid \text { Affine } \mathbb{R}^{3 \times 3} \mathbb{R}^{3} A \quad P::=\text { Cube } \mid \text { Cylinder } \mathbb{N} \mid \ldots \\
& \frac{c \rightarrow_{p} c^{\prime}}{E[c] \rightarrow_{c} E\left[c^{\prime}\right]} \quad \overline{\text { Cube } \rightarrow_{p} \text { Mesh } m_{c u b e}} \\
& \operatorname{map}_{\text {vertex }}(\lambda v \cdot p v+q) m=m^{\prime} \\
& \overline{\text { Affine } p q(\text { Mesh } m) \rightarrow_{p} \text { Mesh } m^{\prime}} \\
& m B o p(o)\left(m_{1}, m_{2}\right)=m^{\prime} \\
& \overline{\text { Binop o }\left(\text { Mesh } m_{1}\right)\left(\text { Mesh } m_{2}\right) \rightarrow p \text { Mesh } m^{\prime}} \\
& \begin{array}{c}
\frac{m \rightarrow_{\Omega} c}{E[\text { Mesh } m] \rightarrow_{s} E[c]} \quad \frac{p \in \Omega_{\text {prim }}(m)}{\text { Mesh } m \rightarrow_{\Omega} p} \\
\frac{\left(m_{1}, m_{2}\right) \in \Omega_{\text {add }}(m)}{\text { Mesh } m \rightarrow_{\Omega} \text { Binop Union }\left(\text { Mesh } m_{1}\right)\left(\text { Mesh } m_{2}\right)} \\
\frac{\left(m_{1}, m_{2}\right) \in \Omega_{\text {sub }}(m)}{\text { Mesh } m \rightarrow_{\Omega} \text { Binop Diff }\left(\text { Mesh } m_{1}\right)\left(\text { Mesh } m_{2}\right)}
\end{array}
\end{aligned}
$$

Fig. 13. Representative cases of small step CAD compilation $\left(\rightarrow_{c}\right.$, left) and synthesis $\left(\rightarrow_{s}\right.$, right) with evaluation contexts and synthesis target language $(E, S)$.

compiled to CAD, many edits which would be tedious or prohibitively difficult at the mesh level become trivial. For example, Figure 12 shows an example of creating a triangle candle holder by starting with a hexagonal one in mesh form, synthesizing $\mathrm{CAD}$, then tweaking the number of sides in Cylinder $(n)$ before printing the desired object.

To develop our synthesis algorithm, we first rephrase our CAD compiler as an equivalent smallstep relation using evaluation contexts and then "flip the arrows" to formalize possible reverse compilations. The resulting synthesis relation captures the fact that many distinct CAD designs may compile down to the same mesh, leading us to introduce a notion of geometric oracles which model the mesh-level heuristics necessary to guide synthesis toward more-editable CAD programs. Following the synthesis relation, our algorithm provides a principled approach to reverse compiling meshes to CAD and enables proving properties of the algorithm as well as clearly delineating the role of heuristics. We assign specifications for the oracles and prove that our synthesis algorithm is correct, i.e., that it preserves semantics. Throughout the section we note key design insights specific to the $\mathrm{CAD}$ domain that focus the heuristics and shrink the search space to speed up synthesis.

\subsection{Specifying Reverse Compilation}

Just as in traditional compilation, translating a CAD program to a mesh loses source-level information. For example, consider the intersection of two cylinders placed side-by-side to form a rounded lozenge in Figure 14a. The resulting mesh only contains fragments of the cylinder primitives the programmer originally specified, yet intuitively we expect synthesis to "figure it out". 
To both make this goal precise and support reasoning by induction on the synthesis search space, we first rephrase our CAD compiler as a small step relation $\rightarrow_{c}$ in Figure 13 which satisfies the property

$$
c \rightarrow_{c}^{*} \text { Mesh } m \Longleftrightarrow \text { compile }(c)=m
$$

At this point, we could specify the target of synthesis as the inverse of $\rightarrow_{c}{ }^{*}$. However, a key component of any mesh-to-CAD synthesis algorithm will be heuristics which infer information lost during compilation. To support reasoning about heuristics' role in synthesis, we instead define the synthesis relation $\rightarrow_{s}$ on the right of Figure 13. The geometric oracle $\Omega_{\text {prim }}$ provides the base case for synthesis by directly recognizing meshes that correspond to a sequence of affine transformations applied to a primitive, while $\Omega_{\text {add }}$ and $\Omega_{\text {sub }}$ indicate when a mesh can be generated by unioning or differencing two "simpler" meshes:

$$
\begin{aligned}
& c \in \Omega_{\text {prim }}(m) \Longrightarrow \llbracket m \rrbracket=\llbracket c \rrbracket \\
& \left(m_{1}, m_{2}\right) \in \Omega_{\mathrm{add}}(m) \Longrightarrow \llbracket m \rrbracket=\llbracket \text { Binop Union }\left(\text { Mesh } m_{1}\right)\left(\text { Mesh } m_{2}\right) \rrbracket \\
& \left(m_{1}, m_{2}\right) \in \Omega_{\text {sub }}(m) \Longrightarrow \llbracket m \rrbracket=\llbracket \text { Binop Diff }\left(\text { Mesh } m_{1}\right)\left(\text { Mesh } m_{2}\right) \rrbracket
\end{aligned}
$$

Assuming these oracle specifications, Mesh $m \rightarrow{ }_{s}^{*} c$ implies compile $(c)=m$. Also, in principle, there exist oracles such that compile $(c)=m$ implies Mesh $m \rightarrow{ }_{s}^{*} c$. However, synthesis does not assume that its input was generated by our compiler; in fact, we intend synthesis to work for meshes obtained from arbitrary sources like online repositories and 3D scanners. In such cases, it is impossible to know what CAD operations (if any) were used to generate the input model.

Synthesis is inherently under-constrained since there is never a unique CAD program that compiles to a given mesh, e.g., for all $c, \llbracket c \rrbracket=\llbracket$ Binop Union $c c \rrbracket$. Furthermore, for any mesh $m$, there is a trivial "complete" synthesis strategy: simply map each face of $m$ to the base of an appropriate inward-facing tetrahedron and take the intersection of the resulting set of tetrahedrons. Such approaches are clearly undesirable as they fail to recover any of the higher-level structure of the original design. To address this, synthesis depends on a ranking function $c_{1} \leq$ edit $c_{2}$ to capture the notion that $c_{2}$ is "more editable" than $c_{1}$. In general, the right choice for $\leq_{\text {edit }}$ will depend on how a user wants to customize a given design, but we have found that program size serves as a good default proxy.

Another challenge is the branching factor in the search space of CAD programs. To help mitigate this issue we restrict the target language of synthesis to the subset $S$ in Figure 13 where only union and difference CSG operations are allowed and are above all affine transformations which in turn are above all primitives. Intuitively, these restrictions are mild since intersections $A \cap B$ can be equivalently expressed as differences $A-(A-B)$ and affine transformations distribute through CSG operations. Two key benefits of this approach are that it focuses the search space by eliminating many equivalent candidates and also suggests a high level strategy composing the primitive, additive, and subtractive oracles. One downside is that CAD programs where affine operations have all been distributed down below CSG operations can be substantially larger; we have found that a simple post-pass to factor out repeated affine operations can often address this issue. Furthermore, once a mesh has been synthesized up to the CAD level, traditional syntax-based synthesis techniques [Bornholt et al. 2016; Phothilimthana et al. 2016; Solar-Lezama 2008] could be applied to further improve editability and optimize other constraints.

Given these definitions and design considerations, we can prioritize some general guidelines for mesh-to-CAD synthesis algorithms with oracles $\Omega$ :

Correct: Synthesis must preserve semantics, $\llbracket \operatorname{synth}_{\Omega}(m) \rrbracket=\llbracket m \rrbracket$.

Useful: Synthesis should strive to generate editable CAD models, i.e., maximize $\leq_{\text {edit }}$.

Predictable: Synthesis should be deterministic in that $\operatorname{synth}_{\Omega}\left(\operatorname{compile}\left(\operatorname{synth}_{\Omega}(m)\right)\right)=\operatorname{synth}_{\Omega}(\mathrm{m})$. 


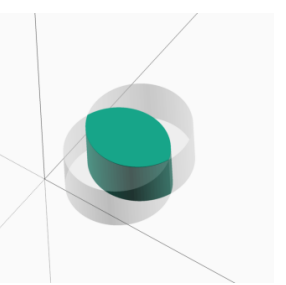

(a) Information lost in compilation.

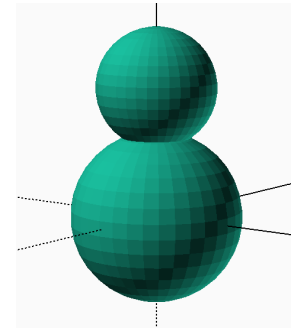

(b) Composition depends on context.

Fig. 14. Figure 14a shows how compiling a CAD to a mesh leads to loss of high level structural information (the fact that the lozenge shape is obtained by intersecting two cylinders). Figure 14b shows how evaluation context can be used to synthesize the union of two spheres.

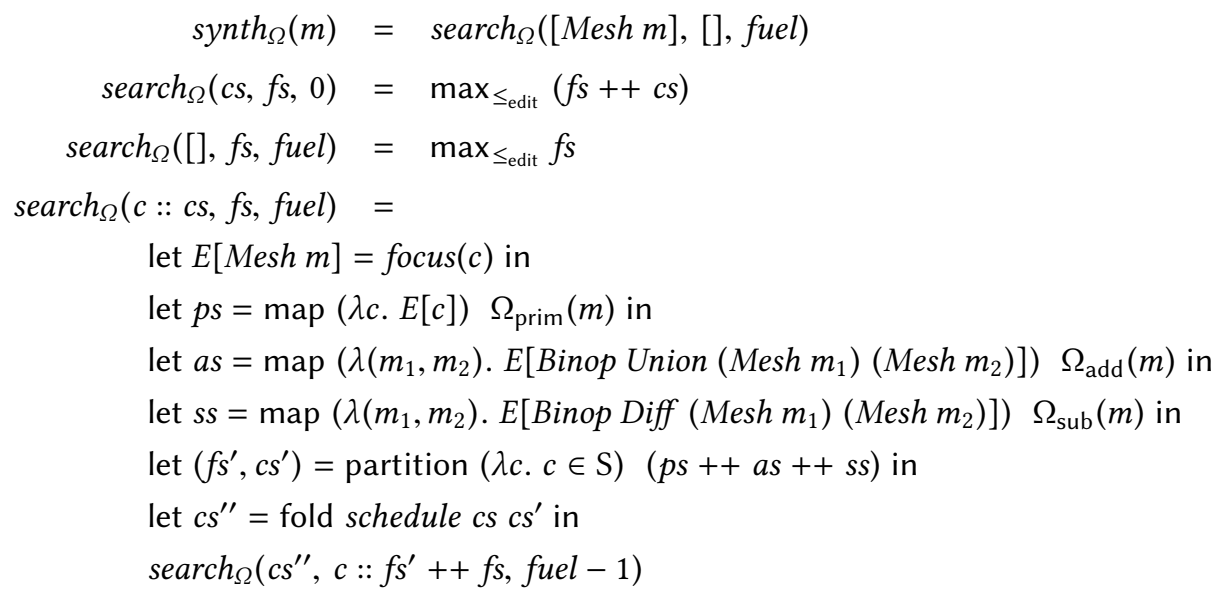

Fig. 15. Core synthesis algorithm.

Complete: $\leq_{\text {edit }}$ should prefer CAD models without embedded meshes (e.g., in $S$ ).

\subsection{Algorithm}

Algorithm 15 shows our synthesis strategy $\operatorname{synth}_{\Omega}()$. The core $\operatorname{search}_{\Omega}$ function maintains a worklist of candidate CAD programs reachable from the input mesh by the $\rightarrow_{s}$ relation. In each iteration, it pops the most promising candidate $c$ from the front of the worklist, focuses on a particular mesh $m$ within $c$, applies oracles in $\Omega$ to $m$ to generate new candidates, and schedules those candidates in the worklist. The algorithm is bounded by a fuel parameter to ensure termination and once it runs out or no candidates remain, search $_{\Omega}$ returns the most editable result according to $\leq_{\text {edit. }}$.

Our synthesis algorithm is designed to be modular: it is straightforward to implement and add new oracles to synthesize a greater variety of CAD programs and control the search by modifying the fuel, focus, schedule, and $\leq_{\text {edit }}$ parameters. Below we describe strategies for effectively implementing geometric oracles and setting these parameters. Since this the goal of this section is to demonstrate the utility of our programming language foundation for $\mathrm{CAD}$, we describe geometric heuristics at a high level. 
$\Omega_{\text {prim. }}$. This oracle recognizes meshes that can be generated by CAD programs in language $A$ from Figure 13, i.e., a sequence of affine transformations applied to a basic primitive. This is straightforward when the mesh corresponds to a primitive in language $P$, but is more challenging for meshes which correspond to rotated, translated, scaled, or skewed versions of a primitive. In such cases, the oracle implementation canonicalizes the input mesh and compares it to canonicalized versions of primitives. If a match is found, it reorients the mesh using the inferred canonicalization parameters and returns the result. We describe canonicalization in more detail in Section 5.4.1.

To implement mesh matching, we designed recognizers for the basic primitives in $P$. These recognizers use geometric properties of the corresponding primitive 3D solids. For example, in order to recognize a cuboid, we check that the mesh is composed of 6 face groups (sets of adjacent faces with equivalent normals), and use the normals of each group to invert any affine transformations which may have been applied to the underlying Cube primitive. To recognize spheroids, we similarly check for (potentially multiple) centroids that have equivalent distances to the faces of the mesh. Similarly, for cylinder and hexagon, we partition the mesh into face groups and use normals to heuristically invert affine transformations.

$\Omega_{\text {add }}$. We experimented with several mesh splitting strategies for this oracle, and ultimately settled on three high level strategies. Disjoint split partitions the mesh by connected components. Convex split identifies a splitting based on rings of coplanar gradient changes. Group split identifies common features between face groups, e.g., being parallel/orthogonal, and separates the mesh along those boundaries.

$\Omega_{\text {sub }}$. Given a mesh $m$, this oracle searches for a bounding mesh that snugly contains $m$ and returns the bound and its difference with $m$. In our current implementation, we limit bounding meshes to those corresponding to CAD primitives. This can be relaxed, and we have observed examples where it would be useful to recursively synthesize more complex bounds, e.g., using convex hull.

Scheduling. To effectively navigate the exponential synthesis search space, the function $\operatorname{search}_{\Omega}$ prioritizes meshes in its worklist deemed more likely to lead to editable CAD programs. As one example from our current implementation of the schedule parameter, we detect when newly generated candidates have meshes of higher overall complexity than where they started and insert such candidates later in the worklist.

focus. In general, a CAD program can match many evaluation contexts. For compilation, only one of these will leave a redex in the context's hole, but for synthesis we may choose any context that places a mesh in the hole. focus examines the full CAD program $c$ and decomposes it to select the most promising mesh. In our current implementation, meshes are selected based on number of face groups and their height in the CAD syntax tree. Focusing has a significant impact on synthesis performance, and more work is needed to characterize the tradeoff between more spending more time to accurately select the most promising mesh and quickly exploring many candidates. We speculate that the tradeoff is actually dynamic in the sense that it often seems to depend on the depth of a mesh within the CAD program.

5.2.1 Context and Sharing. The context of a mesh plays a critical role in synthesis. For example, consider the case of a union of two overlapping spheres ${ }^{2}$ in Figure $14 \mathrm{~b} . \Omega_{\text {add }}$ will use convex splitting to break union into two truncated spheres. No affine transformation of a primitive can match a truncated sphere. Without evaluation context, synthesis would at best be able to return the union of the two meshes of the truncated spheres. However, in context, using full spheres to

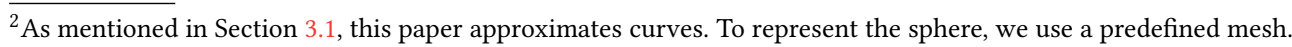


match the truncated spheres works correctly. This is because when we union the complete spheres, the parts of the spheres that are "lost" inside each other are correctly compiled, as we explained in Section 4.

Another important implementation technique is sharing common structure among evaluation contexts to limit memory usage. While the worklist may grow exponentially, we can efficiently represent its contents by reusing common prefixes of each generated evaluation context across the explored programs.

\subsection{Synthesis Correctness}

We briefly sketch the correctness of our synthesis algorithm below and defer evaluating other criteria to the case studies in the following section. One property we rely on is that for any CAD programs $c_{1}$ and $c_{2}$ and evaluation context $E$, $\llbracket c 1 \rrbracket=\llbracket c 2 \rrbracket$ implies $\llbracket E[c 1] \rrbracket=\llbracket E[c 2] \rrbracket$ by the compositional definition of $\llbracket \rrbracket$.

$\llbracket \operatorname{synth}_{\Omega}(m) \rrbracket=\llbracket m \rrbracket$. We first show that Mesh $m \rightarrow_{s}^{*} c$ implies $\llbracket m \rrbracket=\llbracket c \rrbracket$. To get a strong enough induction hypothesis we generalize to prove that $c \rightarrow_{s}^{*} c^{\prime}$ implies $\llbracket c \rrbracket=\llbracket c^{\prime} \rrbracket$, and proceed by induction on the derivation.

Base Case: For 0 steps, $c=c^{\prime}$ and the goal trivially holds.

Inductive case: $c \rightarrow_{s} c^{\prime \prime} \rightarrow_{s}^{*} c^{\prime}$.

By inversion, $c=E\left[\right.$ Mesh $m$ ] and $c^{\prime \prime}=E\left[c^{*}\right]$ for some CAD $c^{*}$.

By the induction hypothesis, $\llbracket c^{\prime \prime} \rrbracket=\llbracket c^{\prime} \rrbracket$.

To show $\llbracket c \rrbracket=\llbracket c^{\prime} \rrbracket$, it is sufficient to establish $\llbracket c \rrbracket=\llbracket c^{\prime \prime} \rrbracket$. This follows from case analysis on the synthesis step, the oracle specifications, and the compositional definition of $\llbracket \rrbracket$.

Now $\llbracket \operatorname{synth}_{\Omega}(m) \rrbracket=\llbracket m \rrbracket$ follows from the invariant that all CAD programs in the worklist during search are reachable under the synthesis relation and the fact that the result of synthesis is drawn from the worklist.

\subsection{Implementation and Challenges}

We implemented our synthesis tool, ReIncarnate to work with the compiler tools we built in Section 4.3. Due to our full functorial design strategy (Section 4.3.2), we were able to implement synthesis as an extension to the existing system by making Synthesis a functor over NumSys, Geometry, Mesh and CAD.

5.4.1 Canonicalization and Re-orientation. In order to match a mesh to a primitive (from a list of predefined primitives) in an arbitrary location and orientation in $3 \mathrm{D}$ space, $\Omega_{\text {prim }}$ performs canonicalization, which is a series of affine transformations applied at the mesh level. This normalizes a mesh with respect to affine transformations-for a mesh, $m$, and an arbitrary sequence of affine transformations, given by a matrix $p$ and translation vector $q$, canonicalize $\mathrm{m}=$ canonicalize $(p m+q)$. The order in which the series of affine transformations in canonicalization are applied is important due to the non-commutativity of affine transformations. The first step in canonicalization is to identify three mutually perpendicular axes of $m$. We do this by identifying three orthogonal directions along which the sum of the areas of the faces is the largest. We call these three axes, $x_{o}, y_{o}$, and $z_{o}$ the object coordinate system. We already know the orthogonal coordinates of the Cartesian coordinate system: $x=(1,0,0), y=(0,1,0)$, and $z=(0,0,1)$ (we call this the world coordinate system). canonicalize solves a linear system of equations using Euler angles [Kim 2013 ] to find three rotations, about $x, y$, and $z$ that can align the world coordinate system to the object coordinate system. Note that this is the opposite of our goal-we want to align the object 
coordinate system to the world coordinate system. canonicalize does this by using the angles obtained from Euler equations, but applying them in the reverse order, and negating the value. If the Euler angles are $r_{x}, r_{y}$, and $r_{z}$, then after canonicalizing with respect to rotation, the new mesh is:

$$
m_{r}=\operatorname{rotateX}\left(-r_{x}\right)\left(\operatorname{rotateY}\left(-r_{y}\right)\left(\operatorname{rotateZ}\left(-r_{z}\right) m\right)\right)
$$

After the axes are aligned, the next step is to scale the mesh to unit dimensions. canonicalize does this by first computing the dimensions of the bounding box of $m_{r},\left(d_{x}, d_{y}, d_{z}\right)$ and scaling it by the reciprocal of the dimensions:

$$
m_{s}=\operatorname{scale}\left(1 / d_{x}, 1 / d_{y}, 1 / d_{z}\right) m_{r}
$$

The final step of canonicalization is to place the center of $m_{s}$ at the origin by translation by finding the bounding box of $m_{s}$ and translating the mid point along each dimension to $(0,0,0)$. If $c_{x}, c_{y}$, and $c_{z}$ are the centers along $x, y$, and $z$ respectively, then

$$
m_{\text {canonicalized }}=m_{t}=\text { translate }\left(-c_{x},-c_{y},-c_{z}\right) m_{s}
$$

Canonicalization is used for primitive matching. Once a primitive $p$ is matched, to synthesize the correct $\mathrm{CAD}, p$ has to be re-oriented to the original location in $3 \mathrm{D}$ space. For this, the algorithm applies the above affine transformations to $p$ in the order:

$$
p^{\prime}=\operatorname{rotateZ}\left(r_{z}\right)\left(\operatorname{rotateY}\left(r_{y}\right)\left(\operatorname{rotateX}\left(r_{x}\right)\left(\operatorname{scale}\left(d_{x}, d_{y}, d_{z}\right) p\right)\right)\right)
$$

The last step is to translate the scaled and rotate primitive, $p^{\prime}$ to the right coordinates. The distance to be translated is the distance between the center of compile $\left(p^{\prime}\right)$ and the center of the original mesh $m:\left(c_{x}^{p^{\prime}}, c_{y}^{p^{\prime}}, c_{z}^{p^{\prime}}\right)-\left(c_{x}^{m}, c_{y}^{m}, c_{z}^{m}\right)$.

$$
\text { poriented }=\text { translate }\left(\left(c_{x}^{p^{\prime}}, c_{y}^{p^{\prime}}, c_{z}^{p^{\prime}}\right)-\left(c_{x}^{m}, c_{y}^{m}, c_{z}^{m}\right)\right) p^{\prime}
$$

The elegance of canonicalization and reorientation for primitives is that it pushes the affine transformations to the leaves of the AST. This makes the rest of synthesis simpler because it saves us from finding canonical orientations of arbitrary binary combinations of CAD programs. This design decision was based on the key insight that while the order of application of affine transformations cannot be changed within themselves, when affine transformations appear with binary transformations, they can be pushed inside the binary operations.

5.4.2 A Concrete Instance of the Synthesis Algorithm. Following is a concrete example of how the ReIncarnate algorithm in Figure 15 works. Consider the mesh $m$ of the model in Figure 16 showing a hexagonal prism in arbitrary location in 3D space. Initially, fuel is greater than 0 and the worklist, cs has one candidate, Mesh $\mathrm{m}$. Consequently, $m$ is the mesh in focus. From Figure 15, we can see that the algorithm will attempt to apply all three oracles to $m$. $\Omega_{\text {prim }}$ will canonicalize the mesh and apply the primitive recognizers. Figure 16 (second figure) shows the canonicalized mesh. Since this already matches with an affine transformed primitive (Cylinder (6)), ps will be a list containing the corresponding $\lambda C A D$ program. This program is shown in Figure 16. Next, the other two oracles, $\Omega_{\text {add }}$ and $\Omega_{\text {sub }}$ will also be applied. $\Omega_{\text {add }}$ will return the original candidate Mesh $m$ since none of the splitting strategies will generate two sub-meshes from $m$. $\Omega_{\text {sub }}$ will return the same result as $\Omega_{\text {prim }}$ since in this case, the snuggest fitting bounding primitive is the affine transformed hexagonal prism. Hence, as will contain Mesh $m$ and $s s$ will contain the same $\lambda$ CAD program as ps. The algorithm will concatenate $p s$, as and $s s$ and remove duplicates before applying the partition function. This will split the list into two parts: $f s^{\prime}$ will contain the $\lambda$ CAD program shown in Figure 16, and $c s^{\prime}$ 

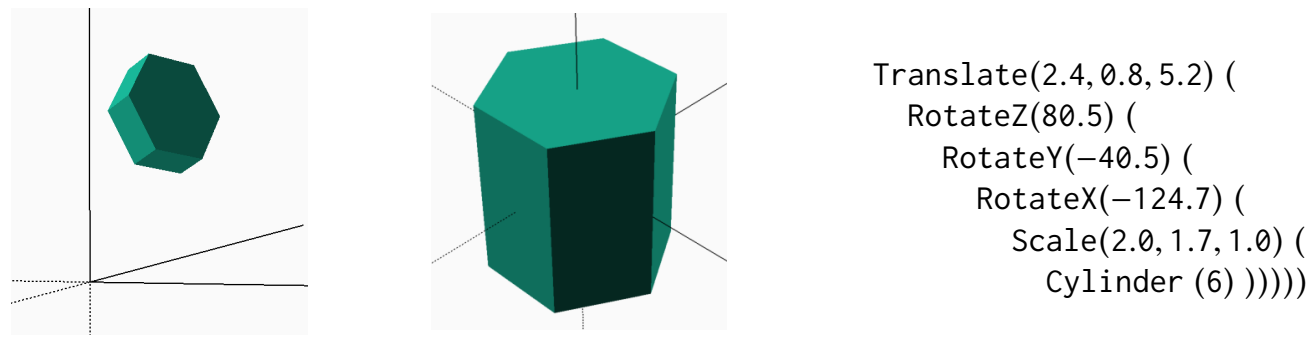

Fig. 16. Canonicalization and $\lambda$ CAD synthesis of arbitrary object in 3D space.

will contain the remaining program, i.e. the result of applying $\Omega_{\text {add }}$, which is the original program, Mesh $\mathrm{m}$. Before scheduling the next mesh to focus on, the algorithm checks whether any of the current candidates were already explored in the previous step. In this case, Mesh $m$ has already been explored, so it will be removed from the list of candidates. At this point, $c s^{\prime \prime}$ thus is an empty list. Therefore, according to the third line in Figure 15 , it will now apply $\max _{\leq_{\text {edit }}}$ to $f$ s. Since $f s$ contains only one $\lambda \mathrm{CAD}$ program (shown in Figure 16), this will be the output of synthesis.

5.4.3 Simplify. We implemented a recursive simplification algorithm, that, given a CAD AST, traverses it to remove redundant nodes. Synthesis can return CAD programs that have redundant nodes in the AST. For example, Binop Union $c c$ is equivalent to $c$ for any CAD program $c$. To remove such redundant nodes, the CAD program is passed on to simplify as the final step. For primitives shapes, simplify simply returns the same node. For nodes that are affine transformations, simplify compiles the child node and if the mesh thus obtained is the same as the mesh with the affine transformation, then simplify removes the affine node. For binary operations, simplify compiles the left child first. If the mesh obtained is the same as the mesh with the binary operation, then simplify returns the left child. If not, it tries the same with the right child. If that fails, then simplify will return the original AST with the binary operation node. Since simplify compiles the child nodes in every recursive step, it can slow down the performance of the synthesis algorithm for very large ASTs. That is why ReIncarnate only uses simplify on the final $\lambda$ CAD program and not at the intermediate stages of synthesis.

\section{CAD SYNTHESIS CASE STUDIES}

We demonstrate three case studies on which we ran ReIncarnate. Two of the case studies are downloaded from Thingiverse and one is our own design (Table 1). They are representative of three of the most common tasks end users of 3D printers typically tend to design for: tools parts, household items, and hobbyist designs [Alcock et al. 2016]. In order to evaluate ReIncarnate's output, we define six tasks:

- scale components of a model, for example a hole inside a bigger part.

- translate components of a model with respect to each other.

- rotate a model as a whole or part of it about one or more axes.

- combine two models or add a new component to an existing model.

- remove a component from a model.

- change \# sides in a regular polygon primitive. This could be for example changing a hexagonal prism to a cylinder or a pentagonal prism.

We give examples of editing tasks from the above categories for each case study to discuss the relative difficulty at both $\lambda \mathrm{CAD}$ and mesh levels. Our overall conclusion is that editing a model after generating $\lambda \mathrm{CAD}$ using ReIncarnate is always easier or the same level of difficulty as editing 
Table 1. Summary of case studies.

\begin{tabular}{|l|l|l|}
\hline Benchmark & Source & Category \\
\hline ICFP & original & hobby \\
\hline Candle holder & Thingiverse & household \\
\hline Hex wrench holder & Thingiverse & tool \\
\hline
\end{tabular}
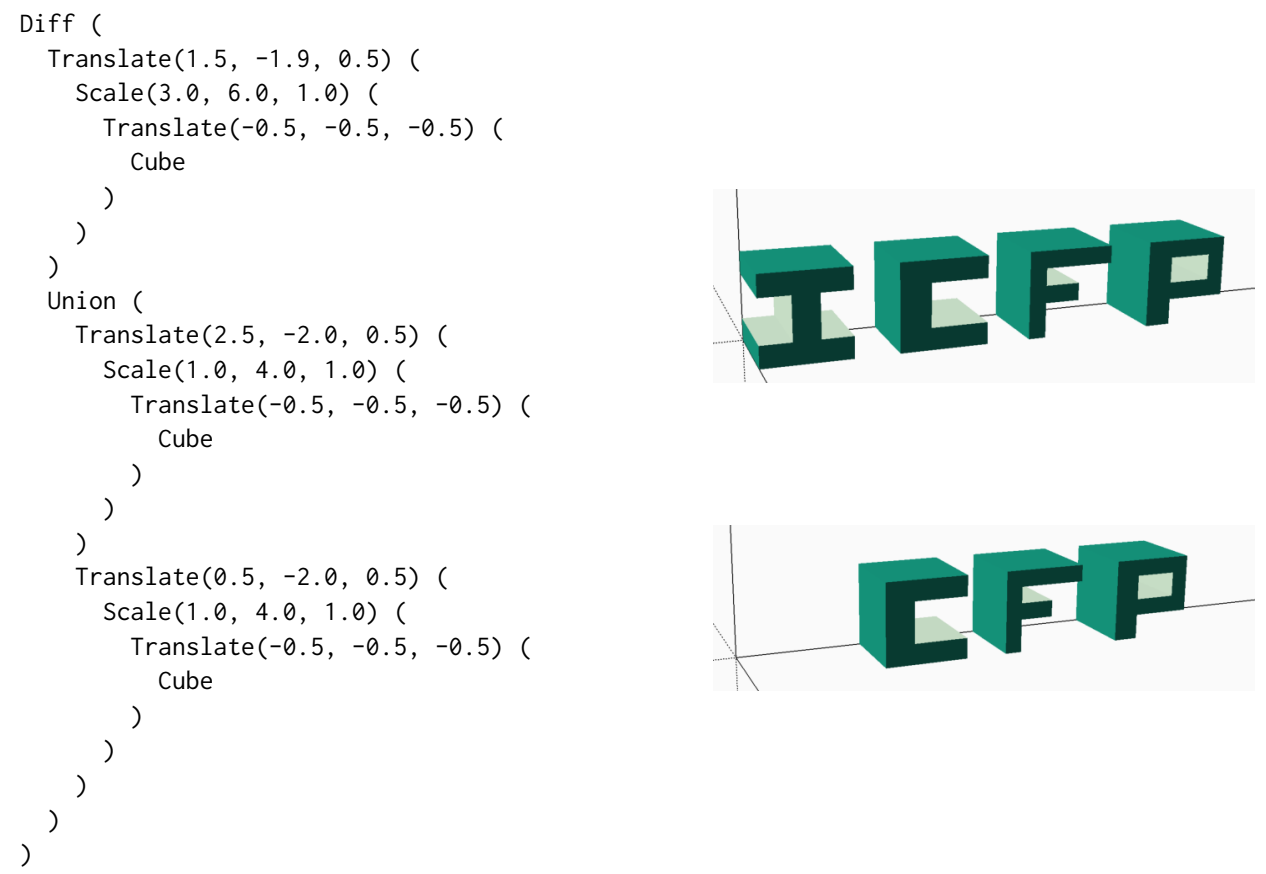

Fig. 17. $\lambda$ CAD program for I synthesized by Relncarnate. Rendered $\lambda$ CAD programs for ICFP and CFP.

the corresponding mesh using mesh editing tools. In several cases, editing the mesh model is as difficult as manually editing the triangular faces which is usually not recommended.

\subsection{ICFP}

This model (shown in Figure 17) was entirely generated by our tools. We designed it in $\lambda \mathrm{CAD}$, compiled it to STL using our compiler (the mesh has approx. 150 faces), and then synthesized a $\lambda$ CAD program using ReIncarnate. The synthesized programs for the individual letters I, $\mathbf{C}, \mathbf{F}$, $\mathbf{P}$ are $25,16,23$, and $23 \mathrm{LOC}$ respectively. The synthesized CAD program for the model ICFP has 89 LOC. Figure 17 shows the $\lambda$ CAD program for I synthesized by ReIncarnate.

- Remove: Consider the task of removing a letter from the model. For example, one can remove the I to model the acronym for Call For Papers shown in Figure 17. Figure 19 shows the $\lambda \mathrm{CAD}$ program for CFP that we obtained by editing the program ReIncarnate synthesized for ICFP. The program is a union of the three letters. Since all the letters are separated, this task should also be relatively easy to perform at the mesh level.

- Translate: Consider an edit where the user wants to increase the spacing between all the letters uniformly. After running ReIncarnate on the mesh, this task is easy: one needs to simply change the translation vector that is used to separate the letters. At the mesh level, 


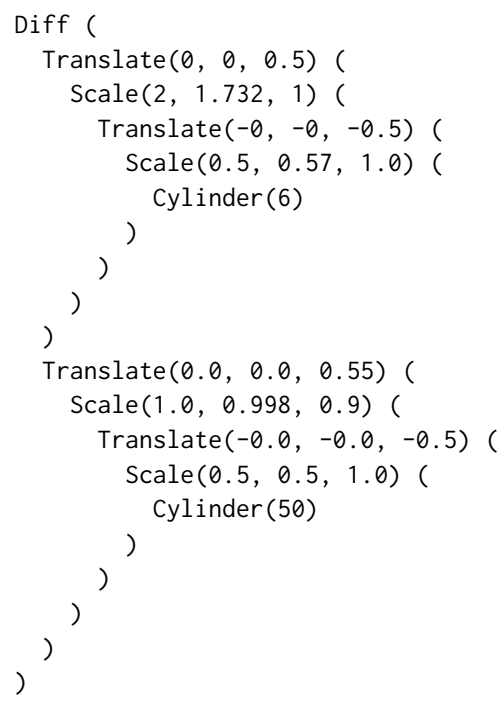

(a) Synthesized $\lambda \mathrm{CAD}$ for the candle holder.

(b) Rendered hex holder.

Fig. 18. Figure 18a shows the $\lambda$ CAD program that Relncarnate synthesized for the candle holder [Thingiverse 2018a]. Figure 18b shows the hex wrench holder from Thingiverse [Thingiverse 2018c].

this seemingly simple task can get confusing and tedious because the user has to drag the letters around to make the spacing uniform.

\subsection{Candle Holder}

Our next example is the candle holder (shown in Figure 2 in Section 2) that we downloaded from Thingiverse [Thingiverse 2018a]. This model is available in STL format, and also in a specific CAD format [Rhinoceros 2018], which is only useful for users who have that CAD package. From a mesh with hundreds of triangular faces, ReIncarnate produced a 20 line CAD program shown in Figure 18a.

- Comments from users [Thingiverse 2018a] indicate that they were unsuccessful in modifying the mesh model to scale it only along $z$-axis using mesh editing tools-it would also change the $x$ and $y$ dimensions. From the user's comment, it seems like even though in theory this task is possible using mesh editing tools [Blender 2018; Meshmixer 2018], it is much more tedious than editing the $\lambda \mathrm{CAD}$ model where it is as simple as adding a scale affine transformation with the right vector (Figure 3).

- Rotating part of the mesh such as the cylindrical hole (about any axis perpendicular to the length of the cylinder) is as difficult as editing the mesh manually because it causes the triangular faces of the hole and the base to intersect with one another, thereby breaking the mesh. This task can be easily done at the $\lambda$ CAD level using RotateX, RotateY.

- Due to the same reason as above, combining the outer polygons to make a bigger base for more than one candle is nearly impossible at the mesh level but very easy at the $\lambda \mathrm{CAD}$ level (using the Union operation).

- Changing the number of sides on the outer polygon is trivial at the $\lambda \mathrm{CAD}$ level (it only requires a single character change to the argument $n$ of Cylinder $(n))$ but as difficult as manually editing the triangular faces at the mesh level. 


\subsection{Hex Wrench Holder}

We were inspired to synthesize the CAD program for a hex wrench holder [Thingiverse 2018c] by a hobbyist maker who downloaded a hex wrench holder mesh and 3D printed it only to find that his hex wrenches did not fit right due to the holes being oriented differently from the shape of his wrenches. The hobbyist tried to used a mesh editing tool to rotate the holes but it was impossible to do this edit because the triangulation of the mesh would break. We synthesized $\lambda C A D$ for the holder using ReIncarnate. The entire $\lambda$ CAD program has 196 LOC (the mesh has over 500 faces). Figure 19 shows a part of the $\lambda \mathrm{CAD}$ program.

- One can use RotateZ $(\theta)$ as shown in Figure 19 to rotate the holes easily in $\lambda \mathrm{CAD}$, but at the mesh level, it is very difficult.

- Scaling the holder holes is yet another task that is very tedious at the mesh level, especially if it is non-uniform. It has the same problem as rotation in that it causes the mesh to break due to intersections between faces.

- Adding or removing a hole are both very easy at the $\lambda \mathrm{CAD}$ level because it requires one to simply scale the cuboidal base of the holder and either subtract another hexagonal prism (adding a hole) or union the model with a hexagonal prism of the right dimensions (removing a hole). These tasks while not impossible at the mesh level, are extremely tedious.

Synthesizing $\lambda \mathrm{CAD}$ for this model opens up directions for future work on CAD synthesis. Figure 18b shows that the holes in the hex holder are all made using the same $\lambda C A D$ primitive, but they are separated by some distance and are scaled differently. ReIncarnate can be extended using classic program synthesis techniques [Bornholt et al. 2016; Phothilimthana et al. 2016] to detect such repetitions and optimize the generated $\lambda \mathrm{CAD}$ programs.

\section{RELATED WORK}

To the best of our knowledge, this is the first work that (1) relates the semantics of CAD and surface meshes using programming-languages techniques and (2) uses program synthesis for reverse engineering $\mathrm{CAD}$ from surface meshes. There are numerous examples from other fields such as human computer interaction, computational geometry, mechanical engineering, computer vision, and design, that have explored 3D models, mesh generation, slicing, and user interfaces to help mitigate current limitations in 3D printing. Below we highlight examples from other communities working on desktop manufacturing. We also provide an overview of state-of-the-art in program synthesis research.

\subsection{Compilers for 3D Printing}

Sutherland's Sketchpad [Sutherland 1964], invented in the 1960s, is one of the first computer-aided design tools. It revolutionized the field of graphical user interfaces and computer-aided simulations. Since then, numerous CAD tools have been developed [Rhinoceros 2018; SketchUp 2018; Solidworks 2018]. Unfortunately, many of these are proprietary and do not provide clear semantics, so it is difficult to reason about them formally.

The core CAD components of $\lambda C A D$ are similar to OpenSCAD [OpenSCAD 2018], which is a popular programmatic CAD tool in the 3D design sharing community [Thingiverse 2018b]. OpenSCAD builds on the CGAL [CGAL 2018] computational geometry library. There are other programmatic CAD languages such as ImplicitCAD [ImplicitCAD 2018] which is implemented in Haskell. ImplicitCAD is similar to OpenSCAD but provides more functionality. Our language and formalism inherits certain restrictions that are also present in OpenSCAD such as lack of direct support for fillets. Unlike our tool, both OpenSCAD and ImplicitCAD lack formal semantics for reasoning about CAD programs. We show that functional programming techniques can be

Proc. ACM Program. Lang., Vol. 2, No. ICFP, Article 99. Publication date: September 2018. 


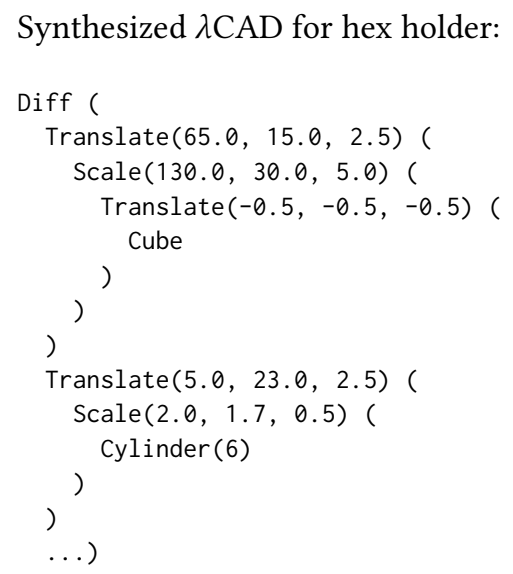

Adding rotation about $\mathrm{Z}$ on line 9:

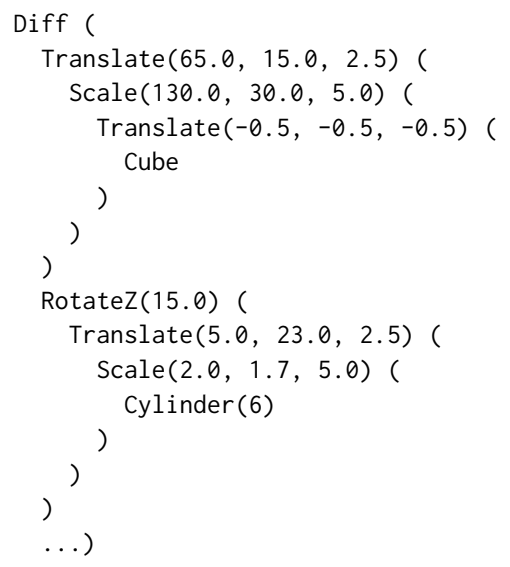

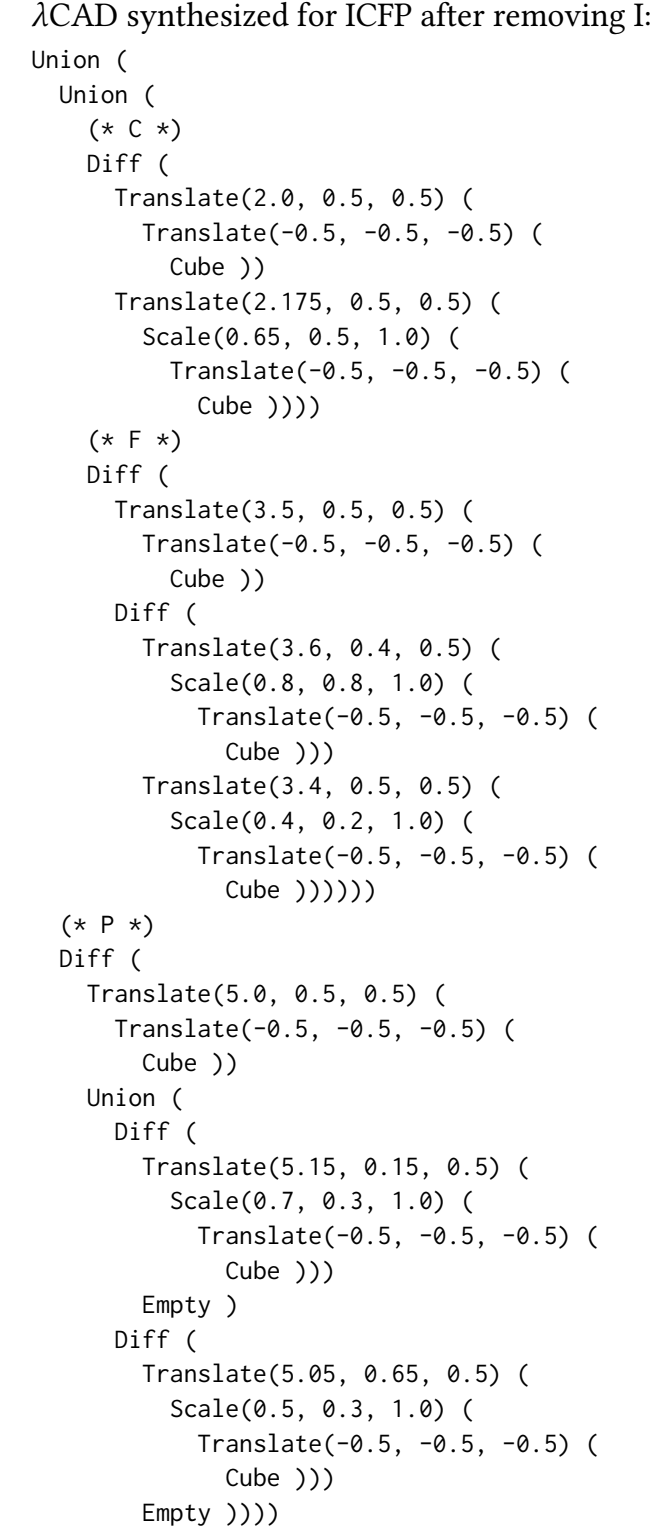
Union 36

Fig. 19. The top left code fragment is part of the $\lambda C A D$ program for the hex holder synthesized by Relncarnate. The bottom left code fragment shows an edit to a hole in the hex wrench holder by rotating the hole by 15 degrees about the $z$-axis on line 9. The $\lambda$ CAD program on the right shows the model for CFP that can be obtained from the $\lambda$ CAD program of ICFP synthesized by Relncarnate by deleting the I. This program is the simply the union of the three letters.

extended to provide a rigorous foundation for reasoning about the implementation and composition of CAD tools. Several projects have investigated 3D-printing performance. WirePrint [Mueller et al. 2014a] and faBrickator [Mueller et al. 2014b] show how non-uniform height slicing and hybrid build approaches can expedite rapid prototyping. OpenFab [Vidimče et al. 2013] is a framework 
for specifying material and texture properties for 3D printing with the help of a domain-specific language. Several projects have developed CAD compilers for unconventional tasks like automated knitting [McCann et al. 2016]. There are also design tools to use 3D printing for modifying existing objects [Chen et al. 2015, 2016] and tools that allow users to correct for measurement errors in CAD models [Kim et al. 2017]. Dumas et al. [Dumas et al. 2015] proposed a texture-synthesis technique that can be used to synthesize texture based on input patterns. Schulz et al. [Schulz et al. 2014] have designed a system that lets casual users design 3D models by example. They first create a database of design templates based on designs by experts, and then let users choose a template and change the parameters. We have previously proposed using PL techniques for 3D-printing, but presented only a preliminary vision without results [Nandi et al. 2017].

\subsection{Analysis of CAD Models}

CAD models can be analyzed before printing to check for structural defects using properties related to materials and geometry [Stava et al. 2012; Zhou et al. 2013]. There are interactive interfaces [McCrae et al. 2014] that let user specify functional parts and provide real-time simulations visualizing stress. Print orientation is a well studied area that focuses on statically analyzing CAD models for maximizing mechanical strength [Umetani and Schmidt 2013]. Other constraints to optimize for could be minimal material usage. Patching existing prints [Teibrich et al. 2015] and analyzing strength properties at the CAD level [Galjaard et al. 2015] are two techniques to accomplish this. Smooth surface finish is another interesting requirement. Delfs et al. [Delfs et al. 2016] developed a tool that achieves smooth surfaces by optimizing the orientation of the part during printing. Krishnamurthy et al. [Krishnamurthy and Levoy 1996] introduced a technique that uses b-splines to smooth models at the mesh level. This work has witnessed tremendous application in the graphics community for rendering $3 \mathrm{D}$ characters.

\subsection{Recreating CAD Models}

There are several tools for reverse engineering CAD from 3D scans [Geomagic Design X 2018; Powershape 2018; SpaceClaim 2018]. The goal of these tools is to help experts manually (re-)create a $\mathrm{CAD}$ design. These tools enhance the traditional CAD workflow primarily by enabling an engineer to "snap" features and dimensions to points from a scan or mesh. Some of these tools also attempt to detect some features and suggest possible primitives (which is similar to the role of $\Omega_{\text {prim }}$ in our synthesis algorithm) or detect coplanar features. Since these tools are proprietary, few details about their implementations are available. These tools are designed to be interactively driven by an expert $\mathrm{CAD}$ engineer and do not produce full CAD programs from meshes.

Thingiverse Customizer [Thingiverse 2018d] is a tool that allows one to modify 3D models uploaded on Thingiverse. It is however only useful for models that include the underlying CAD file. The majority of Thingiverse models do not have an accompanying CAD file, and consist only of mesh-level information in the form of STL files. Customizer cannot reverse engineer CAD programs from the STL meshes, which is the novelty of ReIncarnate.

\subsection{Applications}

Desktop-class 3D printing has started to reach mainstream adoption. Its applications are not only confined to rapid prototyping, and printing tool parts and aesthetic models. The accessibility community has started to use democratized manufacturing to make society more inclusive for people with disabilities [Baldwin et al. 2017; Banovic et al. 2013; Guo et al. 2017; Hofmann et al. 2016b; Hofmann 2015]. The Enable community [The Future 2018] uses 3D printing to print custom prosthesis. This has a huge impact in the developing world where doctors and medical facilities are not available in abundance [Hofmann et al. 2016a]. 


\subsection{Program Synthesis}

Program synthesis is applied to a wide range of applications such as super optimizations for low power spatial architectures [Phothilimthana et al. 2014, 2016], education [Alur et al. 2013] and end-user programming [Wang et al. 2017]. Program synthesis can be inductive or deductive. Inductive syntax-guided program synthesis techniques [Solar-Lezama 2008] fall into the following categories: (1) enumerative search [Udupa et al. 2013], (2) stochastic search [Schkufza et al. 2013], (3) symbolic [Jha et al. 2010]. The main components of these techniques are: a specification that is used to guide the synthesis, a search algorithm to find a candidate program that satisfies the specification, and a feedback mechanism to efficiently prune the search space. In deductive synthesis [Joshi et al. 2002], the specification is a reference implementation and the synthesis algorithm finds an optimal program that is equivalent to the specification on all inputs.

Our synthesis algorithm can be viewed as a reverse-compilation process that starts with a mesh representation of a 3D model as a specification, and searches for a CAD representation of the same. The oracles described in Section 5.2 navigate the search in the right direction. Unlike both traditional deductive and inductive synthesis, neither meshes nor CAD programs take inputs or produce outputs.

\section{FUTURE WORK}

In this section we briefly survey some opportunities for future work to build upon our programminglanguages foundation for 3D printing tools. We focus on numeric and computational geometry challenges to improving mesh-to-CAD synthesis in particular and discuss some directions for exploring further stages of the 3D printing software pipeline.

\subsection{Exact Arithmetic}

One challenge to implementing the semantics described in Sections 3.1 and 3.2 is the need to implement mathematical operations such as square roots and trigonometric functions. Standard floating-point arithmetic and its inherent rounding error is unattractive for reasoning about numerical equivalence [Goldberg 1991; Panchekha et al. 2015]. However, standard exact approaches such as rational arithmetic lack support for trigonometric functions, which are essential in geometry. We have started to investigate the problem of accurate mathematical computation based on the insight that most angles in CAD programs are rational multiples of $\pi$. Such values are algebraic, so can be represented in a splitting field [Artin 2011] of the rational numbers with exact operations and decidable equality/inequality. We can choose a representation of the splitting field where any number is represented by the field size $n$, integer coefficients $a_{i}$ and denominator $d$, representing the value

$$
\frac{1}{d} \sum_{i=0}^{n-1} a_{i} \cos \frac{\pi i}{2 n}
$$

We implemented a prototype of arithmetic operations over these values, including decidable ordering and equality functions, and symbolic square root and arctangent functions, all free from any rounding error. With an overhead of roughly $600 \times$, these exact operations are substantially slower than floating-point operations, but competitive with arbitrary-precision packages. Thanks to our fully-functorial design, users can choose whether to use floating-point or exact arithmetic for their CAD programs, depending on whether speed or high assurance is more important to them. Independently from its benefit to users, this design also allowed us to easily test and debug floating-point code. As a result, though our CAD compiler carries weaker guarantees when run in floating-point mode, we have fairly high confidence that the code is correct. In the future, we 
would like to pursue this direction further and investigate ways to make the number system more complete and performant.

\subsection{Hull}

Computing the convex hull of an object can be added as a built-in unary operator in CAD, and it is a useful one provided by various other tools. The $\lambda \mathrm{CAD}$ implementation already has support for convex hull, but it causes a number of semantic complications that we have not yet fully investigated. Notably, the denotation of the hull operation is not compositional - we need to "inspect" the object whose hull is being computed for things like minimal and maximal points in various dimensions. Semantically this is no problem since we can specify such points with existential quantifiers, but the connection to how hull is compiled is much more subtle. Conversely, our current synthesis procedure never uses hull. Hull is a powerful primitive, and it is unclear in what situations its use helps or hinders producing editable CAD objects.

\subsection{Challenges in Computational Geometry}

Implementing computational geometry involves numerous challenges relating to robustness and performance [Demmel and Hida 2004]. Many of these challenges are due to numerical precision problems [Shewchuk 1997] and as mentioned in Section 8.1, we have started some preliminary investigation in this direction. However, this work's main focus has been on using programming languages to address orthogonal issues of formal specification, correctness and synthesis for CAD. These ideas generalize beyond the details of specific computational geometry techniques and can serve as a foundation for future research.

\subsection{Compiling down to G-code}

Section 2 described that after compiling a CAD program to a mesh, there are two more main compilation steps: slicing and generation of G-code. We have implemented prototypes of both but have not yet proven correctness in terms of semantics. Slicing inherently introduces approximation via discretization since each slice must have a small but nonzero height. Also, not all approaches to slicing produce achievable print strategies due to issues like gravity and the size of the printer.

\section{CONCLUSIONS}

We presented a functional-programming approach to designing and implementing computer-aided design tools. We formalized CAD and surface mesh and provided denotational semantics to both. We implemented a compiler for the semantics and sketched its correctness. We proposed a synthesis technique that uses reverse engineering and geometric oracles to provide CAD programs from surface meshes and showed that it works on real surface meshes downloaded from one of the most popular online repositories for 3D models (Thingiverse). We are optimistic that programminglanguage semantics can continue to provide clarity and functionality in this space, positively affecting an emerging area of computing with potential for mass adoption.

\section{ACKNOWLEDGMENTS}

Thanks to Sarah Chasins, Martin Kellogg, Stuart Pernsteiner, Talia Ringer, Jared Roesch, Ben Sherman, Remy Wang, and Doug Woos for their feedback on early drafts of the paper. We thank John Toman for useful advice on the artifact. We thank Eva Darulova and Max Willsey for numerous stimulating technical conversations. We are grateful to the anonymous reviewers for providing valuable comments. 


\section{REFERENCES}

Celena Alcock, Nathaniel Hudson, and Parmit K. Chilana. 2016. Barriers to Using, Customizing, and Printing 3D Designs on Thingiverse. In Proceedings of the 19th International Conference on Supporting Group Work (GROUP '16). ACM, New York, NY, USA, 195-199. https://doi.org/10.1145/2957276.2957301

Rajeev Alur, Loris D’Antoni, Sumit Gulwani, Dileep Kini, and Mahesh Viswanathan. 2013. Automated Grading of DFA Constructions. In Proceedings of the Twenty-Third International foint Conference on Artificial Intelligence (IFCAI '13). AAAI Press, 1976-1982. http://dl.acm.org/citation.cfm?id=2540128.2540412

M. Artin. 2011. Algebra. Pearson Prentice Hall. https://books.google.com/books?id=S6GSAgAAQBAJ

Mark S. Baldwin, Gillian R. Hayes, Oliver L. Haimson, Jennifer Mankoff, and Scott E. Hudson. 2017. The Tangible Desktop: A Multimodal Approach to Nonvisual Computing. ACM Trans. Access. Comput. 10, 3, Article 9 (Aug. 2017), 28 pages. https://doi.org/10.1145/3075222

Nikola Banovic, Rachel L. Franz, Khai N. Truong, Jennifer Mankoff, and Anind K. Dey. 2013. Uncovering Information Needs for Independent Spatial Learning for Users Who Are Visually Impaired. In Proceedings of the 15th International ACM SIGACCESS Conference on Computers and Accessibility (ASSETS '13). ACM, New York, NY, USA, Article 24, 8 pages. https://doi.org/10.1145/2513383.2513445

Blender. 2018. Blender. (2018). https://www.blender.org/.

James Bornholt, Emina Torlak, Dan Grossman, and Luis Ceze. 2016. Optimizing Synthesis with Metasketches. SIGPLAN Not. 51, 1 (Jan. 2016), 775-788. https://doi.org/10.1145/2914770.2837666

CGAL. 2018. CGAL. (2018). https://www.cgal.org.

Xiang 'Anthony' Chen, Stelian Coros, Jennifer Mankoff, and Scott E. Hudson. 2015. Encore: 3D printed augmentation of everyday objects with printed-over, affixed and interlocked attachments. In Special Interest Group on Computer Graphics and Interactive Techniques Conference, SIGGRAPH '15, Los Angeles, CA, USA, August 9-13, 2015, Posters Proceedings. 3:1. https://doi.org/10.1145/2787626.2787650

Xiang 'Anthony' Chen, Jeeeun Kim, Jennifer Mankoff, Tovi Grossman, Stelian Coros, and Scott E. Hudson. 2016. Reprise: A Design Tool for Specifying, Generating, and Customizing 3D Printable Adaptations on Everyday Objects. In Proceedings of the 29th Annual Symposium on User Interface Software and Technology, UIST 2016, Tokyo, Fapan, October 16-19, 2016. 29-39. https://doi.org/10.1145/2984511.2984512

M. de Berg. 1997. Computational Geometry: Algorithms and Applications. Springer. https://books.google.com/books?id= _vAxRFQcNA8C

P. Delfs, M. T̈ows, and H.-J. Schmid. 2016. Optimized build orientation of additive manufactured parts for improved surface quality and build time. Additive Manufacturing 12, Part B (2016), 314 - 320. https://doi.org/10.1016/j.addma.2016.06.003 Special Issue on Modeling \& Simulation for Additive Manufacturing.

James Demmel and Yozo Hida. 2004. Fast and Accurate Floating Point Summation with Application to Computational Geometry. Numerical Algorithms 37, 1 (01 Dec 2004), 101-112. https://doi.org/10.1023/B:NUMA.0000049458.99541.38

Jérémie Dumas, An Lu, Sylvain Lefebvre, Jun Wu, and Christian Dick. 2015. By-example Synthesis of Structurally Sound Patterns. ACM Trans. Graph. 34, 4, Article 137 (July 2015), 12 pages. https://doi.org/10.1145/2766984

Laurent Fousse, Guillaume Hanrot, Vincent Lefèvre, Patrick Pélissier, and Paul Zimmermann. 2007. MPFR: A Multipleprecision Binary Floating-point Library with Correct Rounding. ACM Trans. Math. Softw. 33, 2, Article 13 (June 2007). https://doi.org/10.1145/1236463.1236468

Salomé Galjaard, Sander Hofman, and Shibo Ren. 2015. New Opportunities to Optimize Structural Designs in Metal by Using Additive Manufacturing. Springer International Publishing, Cham, 79-93. https://doi.org/10.1007/978-3-319-11418-7_6

Geomagic Design X. 2018. Geomagic Design X. (2018). https://www.3dsystems.com/software/geomagic-design-x.

David Goldberg. 1991. What Every Computer Scientist Should Know About Floating-point Arithmetic. Comput. Surveys 23 , 1 (March 1991), 5-48. http://doi.acm.org/10.1145/103162.103163

GrabCAD. 2018. GrabCAD. (2018). https://grabcad.com/.

T. Grimm. 2004. User's Guide to Rapid Prototyping. Society of Manufacturing Engineers.

Anhong Guo, Jeeeun Kim, Xiang ‘Anthony’ Chen, Tom Yeh, Scott E. Hudson, Jennifer Mankoff, and Jeffrey P. Bigham. 2017. Facade: Auto-generating Tactile Interfaces to Appliances. In Proceedings of the 2017 CHI Conference on Human Factors in Computing Systems (CHI '17). ACM, New York, NY, USA, 5826-5838. https://doi.org/10.1145/3025453.3025845

Megan Hofmann, Julie Burke, Jon Pearlman, Goeran Fiedler, Andrea Hess, Jon Schull, Scott E. Hudson, and Jennifer Mankoff. 2016a. Clinical and Maker Perspectives on the Design of Assistive Technology with Rapid Prototyping Technologies. In Proceedings of the 18th International ACM SIGACCESS Conference on Computers and Accessibility (ASSETS '16). ACM, New York, NY, USA, 251-256. https://doi.org/10.1145/2982142.2982181

Megan Hofmann, Jeffrey Harris, Scott E. Hudson, and Jennifer Mankoff. 2016b. Helping Hands: Requirements for a Prototyping Methodology for Upper-limb Prosthetics Users. In Proceedings of the 2016 CHI Conference on Human Factors in Computing Systems (CHI '16). ACM, New York, NY, USA, 1769-1780. https://doi.org/10.1145/2858036.2858340 
Megan Kelly Hofmann. 2015. Making Connections: Modular 3D Printing for Designing Assistive Attachments to Prosthetic Devices. In Proceedings of the 17th International ACM SIGACCESS Conference on Computers and Accessibility (ASSETS '15). ACM, New York, NY, USA, 353-354. https://doi.org/10.1145/2700648.2811323

Kai Hormann and Alexander Agathos. 2001. The Point in Polygon Problem for Arbitrary Polygons. Comput. Geom. Theory Appl. 20, 3 (Nov. 2001), 131-144. https://doi.org/10.1016/S0925-7721(01)00012-8

Nathaniel Hudson, Celena Alcock, and Parmit K. Chilana. 2016. Understanding Newcomers to 3D Printing: Motivations, Workflows, and Barriers of Casual Makers. In Proceedings of the 2016 CHI Conference on Human Factors in Computing Systems (CHI '16). ACM, New York, NY, USA, 384-396. https://doi.org/10.1145/2858036.2858266

ImplicitCAD. 2018. ImplicitCAD. (2018). http://www.implicitcad.org/.

Susmit Jha, Sumit Gulwani, Sanjit A. Seshia, and Ashish Tiwari. 2010. Oracle-guided Component-based Program Synthesis In Proceedings of the 32Nd ACM/IEEE International Conference on Software Engineering - Volume 1 (ICSE '10). ACM, New York, NY, USA, 215-224. https://doi.org/10.1145/1806799.1806833

Rajeev Joshi, Greg Nelson, and Keith Randall. 2002. Denali: A Goal-directed Superoptimizer. In Proceedings of the ACM SIGPLAN 2002 Conference on Programming Language Design and Implementation (PLDI '02). ACM, New York, NY, USA, 304-314. https://doi.org/10.1145/512529.512566

Jeeeun Kim, Anhong Guo, Tom Yeh, Scott E. Hudson, and Jennifer Mankoff. 2017. Understanding Uncertainty in Measurement and Accommodating its Impact in 3D Modeling and Printing. In Proceedings of the 2017 Conference on Designing Interactive Systems, DIS '17, Edinburgh, United Kingdom, June 10-14, 2017. 1067-1078. https://doi.org/10.1145/3064663.3064690

P. Kim. 2013. Rigid Body Dynamics for Beginners: Euler Angles \& Quaternions. CreateSpace Independent Publishing Platform. https://books.google.com/books?id=bJEengEACAAJ

Venkat Krishnamurthy and Marc Levoy. 1996. Fitting Smooth Surfaces to Dense Polygon Meshes. In Proceedings of the 23rd Annual Conference on Computer Graphics and Interactive Techniques (SIGGRAPH '96). ACM, New York, NY, USA, 313-324. https://doi.org/10.1145/237170.237270

James McCann, Lea Albaugh, Vidya Narayanan, April Grow, Wojciech Matusik, Jennifer Mankoff, and Jessica K. Hodgins. 2016. A compiler for 3D machine knitting. ACM Trans. Graph. 35, 4 (2016), 49:1-49:11. https://doi.org/10.1145/2897824. 2925940

James McCrae, Nobuyuki Umetani, and Karan Singh. 2014. FlatFitFab: Interactive Modeling with Planar Sections. In Proceedings of the 27th Annual ACM Symposium on User Interface Software and Technology (UIST '14). ACM, New York, NY, USA, 13-22. https://doi.org/10.1145/2642918.2647388

Autodesk. Meshmixer. 2018. Autodesk. Meshmixer. (2018). http://www.meshmixer.com/.

Stefanie Mueller, Sangha Im, Serafima Gurevich, Alexander Teibrich, Lisa Pfisterer, François Guimbretière, and Patrick Baudisch. 2014a. WirePrint: 3D Printed Previews for Fast Prototyping. In Proceedings of the 27th Annual ACM Symposium on User Interface Software and Technology (UIST '14). ACM, New York, NY, USA, 273-280. https://doi.org/10.1145/2642918. 2647359

Stefanie Mueller, Tobias Mohr, Kerstin Guenther, Johannes Frohnhofen, and Patrick Baudisch. 2014b. faBrickation: Fast 3D Printing of Functional Objects by Integrating Construction Kit Building Blocks. In Proceedings of the SIGCHI Conference on Human Factors in Computing Systems (CHI '14). ACM, New York, NY, USA, 3827-3834. https://doi.org/10.1145/2556288. 2557005

Chandrakana Nandi, Anat Caspi, Dan Grossman, and Zachary Tatlock. 2017. Programming Language Tools and Techniques for 3D Printing. In 2nd Summit on Advances in Programming Languages (SNAPL 2017) (Leibniz International Proceedings in Informatics (LIPIcs)), Benjamin S. Lerner, Rastislav Bodík, and Shriram Krishnamurthi (Eds.), Vol. 71. Schloss DagstuhlLeibniz-Zentrum fuer Informatik, Dagstuhl, Germany, 10:1-10:12. https://doi.org/10.4230/LIPIcs.SNAPL.2017.10

OFF. 2018. OFF Files. (2018). http://www.geomview.org/docs/html/OFF.html.

OpenSCAD. 2018. OpenSCAD. (2018). http://www.openscad.org/.

Pavel Panchekha, Alex Sanchez-Stern, James R. Wilcox, and Zachary Tatlock. 2015. Automatically Improving Accuracy for Floating Point Expressions. In Proceedings of the 36th ACM SIGPLAN Conference on Programming Language Design and Implementation (PLDI '15). ACM, New York, NY, USA, 1-11. https://doi.org/10.1145/2737924.2737959

Phitchaya Mangpo Phothilimthana, Tikhon Jelvis, Rohin Shah, Nishant Totla, Sarah Chasins, and Rastislav Bodik. 2014 Chlorophyll: Synthesis-aided Compiler for Low-power Spatial Architectures. In Proceedings of the 35th ACM SIGPLAN Conference on Programming Language Design and Implementation (PLDI '14). ACM, New York, NY, USA, 396-407. https://doi.org/10.1145/2594291.2594339

Phitchaya Mangpo Phothilimthana, Aditya Thakur, Rastislav Bodik, and Dinakar Dhurjati. 2016. Scaling Up Superoptimization. SIGPLAN Not. 51, 4 (March 2016), 297-310. https://doi.org/10.1145/2954679.2872387

Powershape. 2018. Powershape. (2018). https://www.autodesk.com/products/powershape/overview.

Rhinoceros. 2018. Rhinoceros. (2018). https://www.rhino3d.com/.

Eric Schkufza, Rahul Sharma, and Alex Aiken. 2013. Stochastic Superoptimization. SIGPLAN Not. 48, 4 (March 2013), 305-316. https://doi.org/10.1145/2499368.2451150

Proc. ACM Program. Lang., Vol. 2, No. ICFP, Article 99. Publication date: September 2018. 
Adriana Schulz, Ariel Shamir, David I. W. Levin, Pitchaya Sitthi-amorn, and Wojciech Matusik. 2014. Design and Fabrication by Example. ACM Trans. Graph. 33, 4, Article 62 (July 2014), 11 pages. https://doi.org/10.1145/2601097.2601127

J.R. Shewchuk. 1997. Adaptive precision floating-point arithmetic and fast robust geometric predicates. 18 (10 1997), 305-363.

SketchUp. 2018. SketchUp. (2018). http://www.sketchup.com/.

P. Smid. 2003. CNC Programming Handbook: A Comprehensive Guide to Practical CNC Programming. Industrial Press. https://books.google.com/books?id=JNnQ8r5merMC

Armando Solar-Lezama. 2008. Program Synthesis by Sketching. Ph.D. Dissertation. University of California, Berkeley.

Solidworks. 2018. Solidworks. (2018). http://www.solidworks.com/.

SpaceClaim. 2018. SpaceClaim. (2018). http://www.spaceclaim.com/en/Solutions/ReverseEngineering.aspx.

Ondrej Stava, Juraj Vanek, Bedrich Benes, Nathan Carr, and Radomír Měch. 2012. Stress Relief: Improving Structural Strength of 3D Printable Objects. ACM Trans. Graph. 31, 4, Article 48 (July 2012), 11 pages. https://doi.org/10.1145/2185520.2185544

Ivan E. Sutherland. 1964. Sketch Pad a Man-machine Graphical Communication System. In Proceedings of the SHARE Design Automation Workshop (DAC '64). ACM, New York, NY, USA, 6.329-6.346. https://doi.org/10.1145/800265.810742

Alexander Teibrich, Stefanie Mueller, François Guimbretière, Robert Kovacs, Stefan Neubert, and Patrick Baudisch. 2015. Patching Physical Objects. In Proceedings of the 28th Annual ACM Symposium on User Interface Software \& Technology (UIST '15). ACM, New York, NY, USA, 83-91. https://doi.org/10.1145/2807442.2807467

Enabling The Future. 2018. Enabling The Future. (2018). http://enablingthefuture.org.

Thingiverse. 2018a. Hexagonal Candle Holder. (2018). https://www.thingiverse.com/thing:756968.

Thingiverse. 2018b. Thingiverse. (2018). http://www.thingiverse.com/.

Thingiverse. 2018c. Ultimate 22 Hex-Wrench Holder. (2018). https://www.thingiverse.com/thing:1752602.

Thingiverse. 2018d. Welcome To Customizer. (2018). https://www.thingiverse.com/customizer.

Abhishek Udupa, Arun Raghavan, Jyotirmoy V. Deshmukh, Sela Mador-Haim, Milo M.K. Martin, and Rajeev Alur. 2013. TRANSIT: Specifying Protocols with Concolic Snippets. SIGPLAN Not. 48, 6 (June 2013), 287-296. https://doi.org/10. 1145/2499370.2462174

Nobuyuki Umetani and Ryan Schmidt. 2013. Cross-sectional Structural Analysis for 3D Printing Optimization. In SIGGRAPH Asia 2013 Technical Briefs (SA '13). ACM, New York, NY, USA, Article 5, 4 pages. https://doi.org/10.1145/2542355.2542361

Kiril Vidimče, Szu-Po Wang, Jonathan Ragan-Kelley, and Wojciech Matusik. 2013. OpenFab: A Programmable Pipeline for Multi-material Fabrication. ACM Trans. Graph. 32, 4, Article 136 (July 2013), 12 pages. https://doi.org/10.1145/2461912. 2461993

Chenglong Wang, Alvin Cheung, and Rastislav Bodik. 2017. Synthesizing Highly Expressive SQL Queries from Input-output Examples. SIGPLAN Not. 52, 6 (June 2017), 452-466. https://doi.org/10.1145/3140587.3062365

Qingnan Zhou, Julian Panetta, and Denis Zorin. 2013. Worst-case Structural Analysis. ACM Trans. Graph. 32, 4, Article 137 (July 2013), 12 pages. https://doi.org/10.1145/2461912.2461967

Paul Zimmermann. 2010. Reliable Computing with GNU MPFR. In Proceedings of the Third International Congress Conference on Mathematical Software (ICMS'10). Springer-Verlag, Berlin, Heidelberg, 42-45. http://dl.acm.org/citation.cfm?id= 1888390.1888400 